\title{
Rhodium-Catalyzed Methylenation of Aldehydes.
}

\section{Hélène Lebel* and Valérie Paquet.}

Département de chimie, Université de Montréal, Montréal, Québec, Canada, H3C 3J7

\section{Supporting Information}

Table 1. Rhodium-Catalyzed Methylenation of Cinnamaldehyde. 3

Table 2. Solvent Effect on the Rhodium-Catalyzed Methylenation of 6-(tert-Butyldimethylsilyloxy)-1-pentanal with Trimethylsilyldiazomethane 4

Table 3. Rhodium-Catalyzed Methylenation of 6-(tert-Butyldimethylsilyloxy)-1-pentanal with Various Trimethylsilyldiazomethane Solutions.

Table 4. Rhodium-Catalyzed Methylenation of Aldehydes with commercial solution and freshly prepared trimethylsilyldiazomethane solution.

General Information .7

Trimethylsilyldiazomethane Synthesis. 8

Phosphine Synthesis. .8

Olefination Procedures A to C .11

Characterization of Alkene Products.. .11

GC Trace of Various Trimethylsilyldiazomethane Solutions. .23 
An investigation of other rhodium complexes shows that the requisite catalyst may also be generated in-situ from a number of rhodium sources, due to the presence of an excess of triphenylphosphine in the reaction mixture (Table 1). For instance, rhodium (III) chloride hydrate ${ }^{1}$ may react directly with triphenylphosphine under the methylenation reaction conditions. Since the formation of the Wilkinson's catalyst does not proceed at room temperature, the reaction run at 50 ${ }^{\circ} \mathrm{C}$ (entry 2). Alternatively, we can also preformed in-situ the desired catalyst from rhodium (III) chloride hydrate and triphenylphosphine at $50{ }^{\circ} \mathrm{C}$, and then cooled the reaction mixture to room temperature, prior to the addition of the substrate and the other reagents (2-propanol and trimethylsilyldiazomethane) required to carry out the methylenation reaction (entry 3). Rhodium (I) dimer complexes are also suitable precursors for the in-situ generation of the Wilkinson's catalyst. While the reaction of chloro[1,5-cyclooctadiene] rhodium (I) dimer and triphenylphosphine also proceeded only at $50{ }^{\circ} \mathrm{C}$ (entries 4-6), the use of a more labile diene, such as norbornadiene allowed the reaction to occur at room temperature (entries 7-8). ${ }^{2}$ In comparison, the corresponding cationic complexes derived from silver tetrafluoroborate and sodium tetraphenylborate were less reactive and the olefination reaction took place only at $50{ }^{\circ} \mathrm{C}$ (entries 9-12). Formally the rhodium (0) complex $\mathrm{Rh}(\mathrm{NO})\left(\mathrm{PPh}_{3}\right)_{3}$ seems also to be an active catalyst, although a very long latent period was observed prior to the beginning of the olefination reaction (entry 13). This latent period and the many color variations of the reaction mixture are consistent with the hypothesis that a redox process initially occurs, leading to an active rhodium (I) species. In contrast, no reaction was observed with the rhodium (II) acetate dimer, although this complex has found many applications in carbene chemistry (entry 14). ${ }^{3}$

\footnotetext{
${ }^{1}$ Due to solubility issues, it is required to use rhodium (III) chloride hydrate (instead of rhodium (III) chloride anhydrous) to prepare the chlorotris(triphenylphosphine)rhodium (I).

${ }^{2}$ The formation of the desired chlorotris(triphenylphosphine)rhodium (I) was monitored by phosphorus NMR. 3 (a) Paulissen, R.; Reimlinger, H.; Hayez, E.; Hubert, A. J.; Teyssie, P. Tetrahedron Lett. 1973, 2233-2236. (b) Doyle, M. P.; McKervey, M. A.; Ye, T. Modern Catalytic Methods for Organic Synthesis with Diazo Compounds: From Cyclopropanes to Ylides.; Wiley: New York, 1998. (c) Timmons, D. J.; Doyle, M. P. J. Organomet. Chem. 2001, 617, 98-104. (d) Doyle, M. P.; Ren, T. Prog. Inorg. Chem. 2001, 49, 113-168. (e) Lebel, H.; Marcoux, J. F.; Molinaro, C.; Charette, A. B. Chem. Rev. 2003, 103, 977-1050. (f) Merlic, C. A.; Zechman, A. L. Synthesis 2003, 1137-1156.
} 
Table 1. Rhodium-Catalyzed Methylenation of Cinnamaldehyde.

\begin{tabular}{|c|c|c|}
\hline Entry & Catalyst & Conv. ${ }^{a}$ \\
\hline 1 & $\mathrm{RhCl}\left(\mathrm{PPh}_{3}\right)_{3} ; 25{ }^{\circ} \mathrm{C}, 0.5 \mathrm{~h}$ & $\geq 98 \%$ \\
\hline 2 & $\mathrm{RhCl}_{3} \cdot \mathrm{H}_{2} \mathrm{O} ; 50{ }^{\circ} \mathrm{C}, 1 \mathrm{~h}$ & $\geq 98 \%$ \\
\hline 3 & $\mathrm{RhCl}_{3} \cdot \mathrm{H}_{2} \mathrm{O} ; 25^{\circ} \mathrm{C}, 1 \mathrm{~h}^{b}$ & $\geq 98 \%$ \\
\hline 4 & {$[\mathrm{Rh}(\mathrm{COD}) \mathrm{Cl}]_{2} ; 25^{\circ} \mathrm{C}, 4 \mathrm{~h}$} & $19 \%$ \\
\hline 5 & {$[\mathrm{Rh}(\mathrm{COD}) \mathrm{Cl}]_{2} ; 25^{\circ} \mathrm{C}, 0.5 \mathrm{~h}^{b}$} & $\geq 98 \%$ \\
\hline 6 & {$[\mathrm{Rh}(\mathrm{COD}) \mathrm{Cl}]_{2} ; 50^{\circ} \mathrm{C}, 1 \mathrm{~h}$} & $\geq 98 \%$ \\
\hline 7 & {$[\mathrm{Rh}(\mathrm{NBD}) \mathrm{Cl}]_{2} ; 25^{\circ} \mathrm{C}, 1.5 \mathrm{~h}$} & $\geq 98 \%$ \\
\hline 8 & {$[\mathrm{Rh}(\mathrm{NBD}) \mathrm{Cl}]_{2} ; 50{ }^{\circ} \mathrm{C}, 0.5 \mathrm{~h}$} & $\geq 98 \%$ \\
\hline 9 & {$\left[\mathrm{Rh}(\mathrm{COD})_{2}\right]^{+} \mathrm{BF}_{4}^{-} ; 25^{\circ} \mathrm{C}, 16 \mathrm{~h}$} & $\leq 5 \%$ \\
\hline 10 & {$\left[\mathrm{Rh}(\mathrm{COD})_{2}\right]^{+} \mathrm{BF}_{4}^{-} ; 50^{\circ} \mathrm{C}, 0.3 \mathrm{~h}$} & $\geq 98 \%$ \\
\hline 11 & {$[\mathrm{Rh}(\mathrm{COD})]^{+} \mathrm{BPh}_{4}^{-} ; 25^{\circ} \mathrm{C}, 16 \mathrm{~h}$} & $\leq 5 \%$ \\
\hline 12 & {$[\mathrm{Rh}(\mathrm{COD})]^{+} \mathrm{BPh}_{4} ; 50{ }^{\circ} \mathrm{C}, 3 \mathrm{~h}$} & $\geq 98 \%$ \\
\hline 13 & $\mathrm{Rh}(\mathrm{NO})\left(\mathrm{PPh}_{3}\right)_{3} ; 25^{\circ} \mathrm{C}, 8 \mathrm{~h}$ & $\geq 98 \%$ \\
\hline 14 & $\mathrm{Rh}_{2}(\mathrm{OAc})_{4} ; 25^{\circ} \mathrm{C}, 16 \mathrm{~h}$ & $\leq 5 \%$ \\
\hline
\end{tabular}


Table 2. Solvent Effect on the Rhodium-Catalyzed Methylenation of 6-(tertButyldimethylsilyloxy)-1-pentanal with Trimethylsilyldiazomethane.

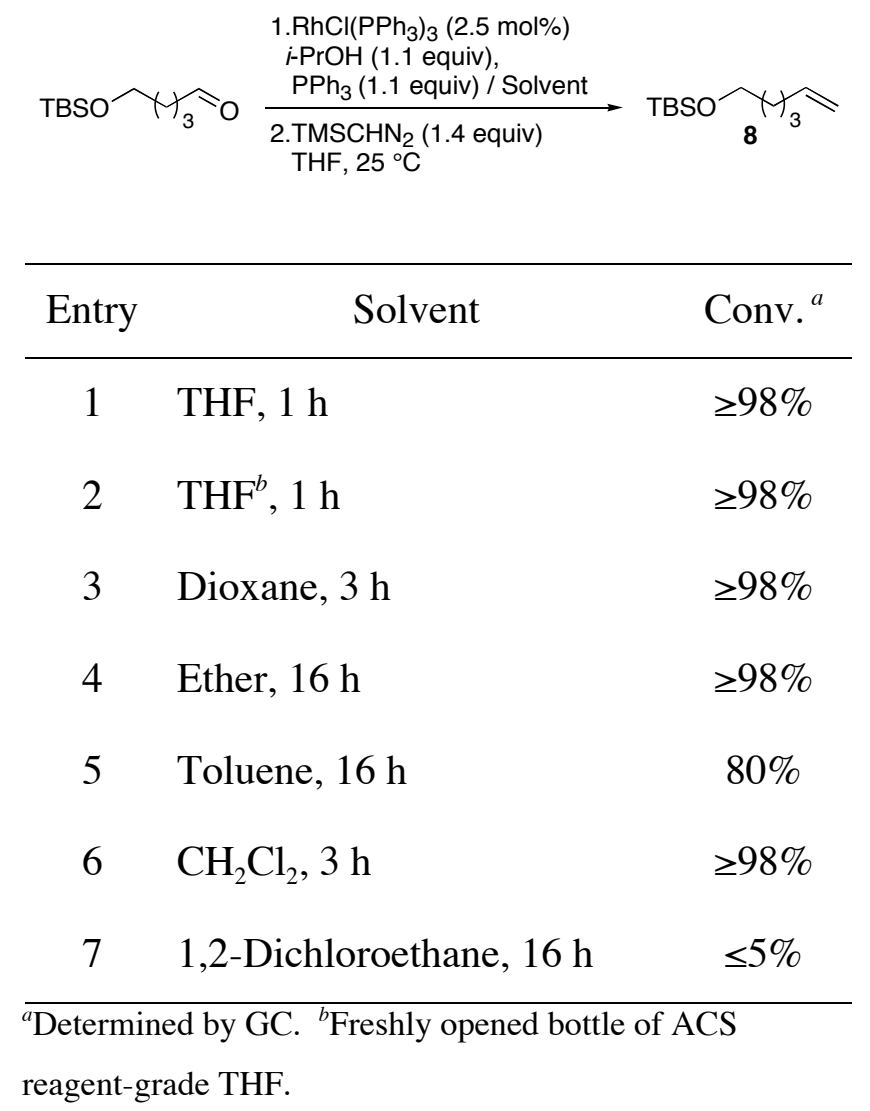


Table 3. Rhodium-Catalyzed Methylenation of 6-(tert-Butyldimethylsilyloxy)-1-pentanal with Various Trimethylsilyldiazomethane Solutions.

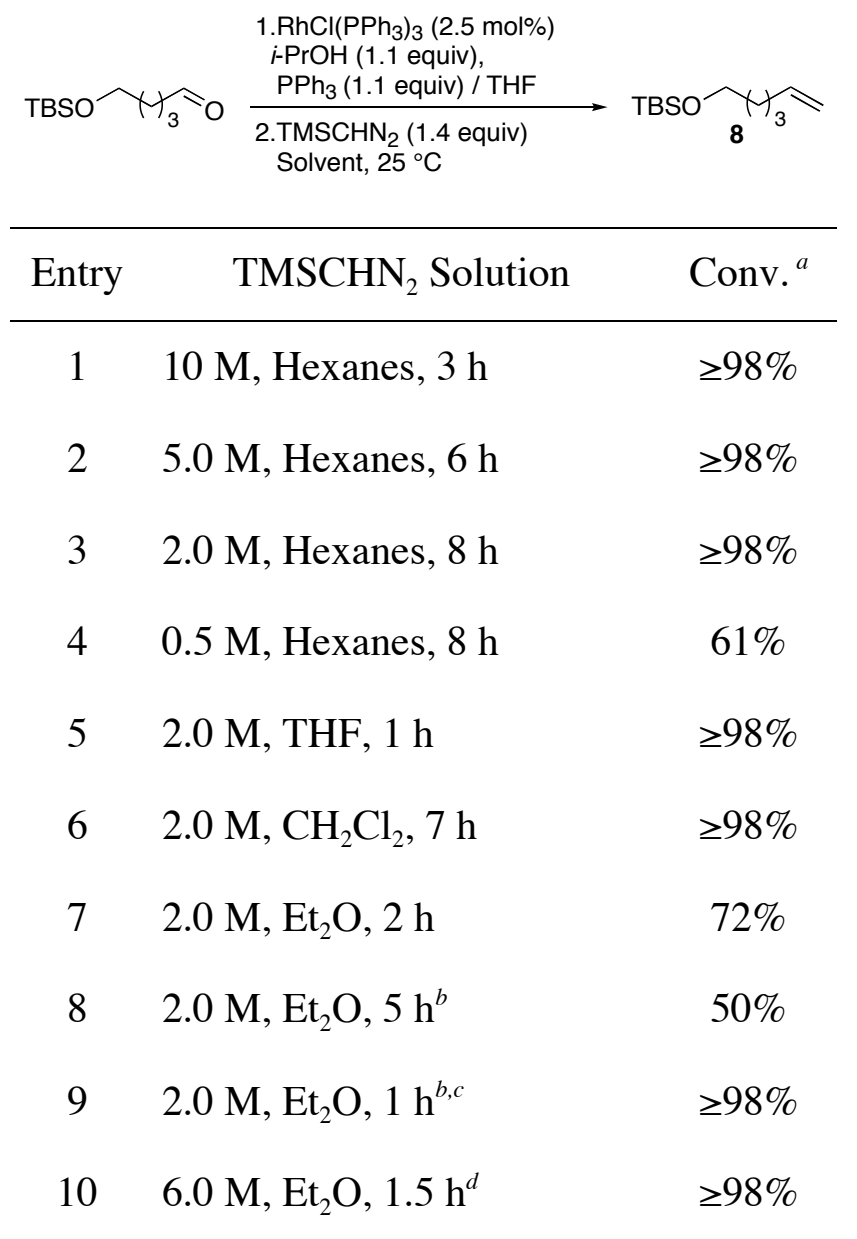

${ }^{a}$ Determined by GC. ${ }^{b}$ Solution from Aldrich. ${ }^{c} 1.7$ equiv. were used. ${ }^{d}$ Solution from Aldrich that was concentrated. 
Table 4. Rhodium-Catalyzed Methylenation of Aldehydes with commercial solution and freshly prepared trimethylsilyldiazomethane solution.

\begin{tabular}{|c|c|c|c|c|}
\hline \multirow{2}{*}{\multicolumn{2}{|c|}{$\mathrm{R} \curvearrowright 0$}} & $\begin{array}{l}\text { 1. } \mathrm{RhC} \\
i-\mathrm{PrOH}\end{array}$ & $\begin{array}{l}5 \mathrm{~mol} \%) / \mathrm{THF} \\
\mathrm{PPh}_{3}(1.1 \text { equiv) }\end{array}$ & \multirow{2}{*}{$\mathrm{R} \curvearrowright$} \\
\hline & & 2. TMS &, $25^{\circ} \mathrm{C}$ & \\
\hline Entry & & Product & $\begin{array}{l}\mathrm{TMSCHN}_{2}{ }^{a} \\
2.0 \mathrm{M} / \text { ether } \\
1.7 \text { equiv. }\end{array}$ & $\begin{array}{c}\mathrm{TMSCHN}_{2} \\
2.0 \mathrm{M} / \mathrm{THF} \\
1.4 \text { equiv. }\end{array}$ \\
\hline 1 & TBSC & & $\begin{array}{l}77 \% \text { y. } 1 \mathrm{~h} \\
(85 \% \text { y. } 2 \mathrm{~h})^{b}\end{array}$ & $87 \%$ y. $1 \mathrm{~h}$ \\
\hline 2 & & & $83 \%$ y. $1 \mathrm{~h}$ & $88 \%$ y. $0.5 \mathrm{~h}$ \\
\hline 3 & & & $87 \%$ y. $1 \mathrm{~h}$ & $93 \%$ y. $1 \mathrm{~h}$ \\
\hline 4 & & & $\begin{array}{l}79 \% \text { y. } 5 \mathrm{~h} \\
(94 \% \text { ee) }\end{array}$ & $\begin{array}{l}86 \% \text { y. } 4 \mathrm{~h} \\
(92 \% \text { ee) }\end{array}$ \\
\hline 5 & & & $87 \%$ y. 3 h & $87 \%$ у. 3 h \\
\hline
\end{tabular}

${ }^{a}$ Commercially available solution. ${ }^{\text {aThe }}$ concentrated $5 \mathrm{M}$ solutionwas used. 
General: Unless otherwise noted, all non-aqueous reactions were performed under an oxygen-free atmosphere of argon with rigid exclusion of moisture from reagents and glassware. The solvents were dried using standard methods prior to use. $\mathrm{RhCl}\left(\mathrm{PPh}_{3}\right)_{3}$ is commercially available, but was prepared from $\mathrm{RhCl}_{3} \bullet 3 \mathrm{H}_{2} \mathrm{O}$ and $4 \mathrm{PPh}_{3}$ according to the literature. ${ }^{4}$ Methanol, ethanol, 2-propanol, benzyl alcohol, 2-butanol and 2-methyl-1-propanol was distilled over calcium hydride. All the cationic complexes of rhodium (I) were prepared according to the literature procedure, ${ }^{5}$ except for the preparation of $\left[\mathrm{Rh}(\mathrm{COD})_{2}{ }^{+} \mathrm{BF}_{4}^{-}\right] .{ }^{6}$ The commercially available aldehydes were purified using standard methods prior to use. The following compounds were received from commercial suppliers (Aldrich Chemical Co, Strem Chemicals Inc.) and used without further purification: triphenylphosphine, tris(4-methoxyphenyl)phosphine, tributylphosphine, 4-(diphenylphosphino) benzoic acid, tris(4-fluorophenyl)phosphine, tris(4-trifluoromethylphenyl)phosphine, 2-methyl-2propanol, phenol, chloro(1,5-cyclooctadiene) rhodium (I) dimer, chloronorbornadiene rhodium (I) dimer. Analytical thin layer chromatography (TLC) was performed using EM Reagent $0.25 \mathrm{~mm}$ silica gel $60-\mathrm{F}$ plates. Visualization of the developed chromatogram was performed by UV absorbance, aqueous cerium molybdate, ethanolic phosphomolybdic acid, or aqueous potassium permanganate. Flash chromatography was performed using EM Silica Gel 60 (230-400 mesh) with the indicated solvent system. Optical rotations were measured on a Perkin-Elmer 341 digital polarimeter at $589 \mathrm{~nm}$. Data are reported as follows: [a] $]_{1}^{\text {temp. }}$, concentration $(c \mathrm{~g} / 100 \mathrm{~mL})$, and solvent. Infrared spectra were recorded on a Perkin Elmer Spectrum One FTIR spectrometer equipped with a Golden Gate Diamond ATR and are reported in reciprocal centimeters $\left(\mathrm{cm}^{-1}\right)$. Only the most important and relevant frequencies are reported. ${ }^{1} \mathrm{H}$ NMR spectra were recorded in deuteriochloroforme, unless otherwise noted, on a Bruker AV-400, a Bruker ARX-400, a Bruker AMX-300 or a Bruker AV-300 spectrometers (400, 400, 300 and $300 \mathrm{MHz}$ respectively). Chemical shifts are reported in ppm on the $\mathrm{d}$ scale from an internal standard of residual chloroform $(7.27 \mathrm{ppm})$. Data are reported as follows: chemical shift, multiplicity $(\mathrm{s}=$ singlet, $\mathrm{d}=$ doublet, $\mathrm{t}=$ triplet, $\mathrm{q}=$ quartet, $\mathrm{qn}=$ quintet, $\mathrm{m}=$ multiplet and $\mathrm{br}=$ broad), coupling constant in $\mathrm{Hz}$, integration. ${ }^{13} \mathrm{C}$ NMR spectra were recorded in deuteriochloroforme, unless otherwise noted, on a Bruker AV400, a Bruker ARX-400, a Bruker AMX-300 or a Bruker AV-300 spectrometers $(100,100,75$ and $75 \mathrm{MHz}$ respectively) with complete proton decoupling. Chemical shifts are reported in ppm from the central peak of deuteriochloroform $(76.9 \mathrm{ppm})$ on the $\mathrm{d}$ scale. ${ }^{19} \mathrm{~F}$ NMR spectra were recorded in deuteriochloroforme, unless otherwise noted, on a Bruker ARX-400 spectrometer $(376,5 \mathrm{MHz})$ with complete proton decoupling. Chemical shifts are reported in ppm from the peak of trifluoroacetic acid $(-78.5 \mathrm{ppm})$ on the $\square$ scale. ${ }^{31} \mathrm{P}$ NMR spectra were recorded in deuteriochloroforme, unless otherwise noted, on a Bruker ARX-400 spectrometer $(162 \mathrm{MHz})$ with complete proton decoupling. Chemical shifts are reported in ppm from the peak of $85 \% \mathrm{H}_{3} \mathrm{PO}_{4}$ in $\mathrm{D}_{2} \mathrm{O}(0 \mathrm{ppm})$ on the $\square$ scale. Mass spectra were obtained on a KRATOS MS-50 TC TA (FAB) or a Micromass Autospec-TOF (MAB) high resolution magnetic sector mass spectrometer by the Centre régional de spectrométrie de masse de l'Université de Montréal. The elementary analyses were performed by the Laboratoire d'analyse élémentaire de l'Université de Montréal or by the Canadian Microanalytical Service ltd. High performance liquid chromatography (HPLC) analyses were performed on a Hewlett Packard 1100 Series quaternary gradient pump with diode-array detector interfaced with HP Chemstation software. Values for enantiomeric excess were determined using a Chiracel OJ column. Data are reported as follows: column type, flow, solvent used, and retention time $\left(\mathrm{t}_{\mathrm{r}}\right)$. Analytical gas chromatography (GLC) was carried out on a Hewlett Packard 6890 series gas chromatograph equipped with a split mode capillary injector and a flame ionization detector. Unless otherwise noted, injector and detector temperatures were $250{ }^{\circ} \mathrm{C}$ and the carrier gas was hydrogen. Data are reported as follows: column type, oven temperature, and retention time $\left(\mathrm{t}_{\mathrm{r}}\right)$.

\footnotetext{
4 Osborn J. A.; Wilkinson, G. Inorg. Synth. 1990, 28, 77-79.

5 Osborn, J. A.; Schrock, R. R. J. Am. Chem. Soc. 1971, 93, 2397-2407.

6 Schenck, T. G.; Downes, J. M.; Milne, C. R. C.; Mackenzie, P. B.; Boucher, T. G.; Whelan, J.; Bosnich, B. Inorg. Chem. 1985, 24, 2334-2337.
} 
Synthesis of Trimethylsilyldiazomethane. ${ }^{7,{ }^{7}}$ A dry, $1 \mathrm{~L}$, three-necked round-bottomed flask is equipped with a $250 \mathrm{~mL}$ pressure-equalizing dropping funnel, a stirring bar and two septum. The apparatus is flame-dry under argon. When the system is cooled at room temperature, a solution of diphenylphosphorazidate ${ }^{9}(46.0 \mathrm{~g}, 167 \mathrm{mmol})$ in $200 \mathrm{~mL}$ of anhydrous diethyl ether is transfered to the flask via a cannula. The ether solution of the Grignard reagent ${ }^{4}(200 \mathrm{~mL}, 200 \mathrm{mmol})$ is measured in a graduate cylinder via cannula under argon and then transferred to the dropping funnel. The flask is cooled with a dry-ice/acetone bath. When the internal temperature reaches -10 ${ }^{\circ} \mathrm{C}$ (measured with an electronic thermometer), the Grignard reagent is added dropwise at such a rate that the internal temperature is maintained below $0{ }^{\circ} \mathrm{C}$ (some ether can be added for better stirring). After the addition is completed, the reaction is stirred for 2 hours with an ice bath. The funnel is replaced by a septum and the flask is then placed in the fridge for 14 hours. The reaction mixture is opened to air and cooled again to $-15^{\circ} \mathrm{C}$ with a dry-ice/acetone bath. Water $(50 \mathrm{~mL})$ is carefully added dropwise at such a rate that the internal temperature is maintained below $0{ }^{\circ} \mathrm{C}$ (stirring can be done manually). The reaction mixture is filtered with a Buckner filter and the white solid is washed with ether $(100 \mathrm{~mL})$. The yellow filtrate is washed with water $(100 \mathrm{~mL})$ and dried over $\mathrm{MgSO}_{4}$. The filtrate is placed in a $1 \mathrm{~L}$ round-bottomed flask with a magnetic stirrer and equipped with a distillation apparatus (the vigreux column must be between 5 to $10 \mathrm{~cm}$ ). The solution is slowly distilled under argon until no more volatile comes over (oil bath temperature below $50{ }^{\circ} \mathrm{C}$ ). The oil bath is removed and the distillation apparatus is replaced by a "U" glass tube connected to a $250 \mathrm{~mL}$ round-bottomed flask placed in a dry-ice/acetone bath. The solution is transferred under vacuum $(0.1 \mathrm{mmHg})$. At the end, the oil bath at $50{ }^{\circ} \mathrm{C}$ can be used to transfer the remaining trimethylsilyldiazomethane. The yellow solution is dried over $\mathrm{Na}_{2} \mathrm{SO}_{4}$ at room temperature. After the filtration in a $100 \mathrm{~mL}$ flask, the solution is concentrated slowly, until the vapors reached $80{ }^{\circ} \mathrm{C}$. The remaining trimethylsilyldiazomethane can be used as it is and transfer in an amber bottle under argon (the product is light sensitive).

Purification from a commercially available solution. The Aldrich solution of trimethylsilyldiazomethane in ether $(25.0 \mathrm{~mL}, 50.0 \mathrm{mmol})$ was concentrated into a $6 \mathrm{M}$ solution. ${ }^{10}$ The distillation was performed over a period of 2 hours under argon with a vigreux column of $5 \mathrm{~cm}$ and the oil bath temperature has raised from $40{ }^{\circ} \mathrm{C}$ to $100{ }^{\circ} \mathrm{C}$.

\section{Synthesis of Phosphines}

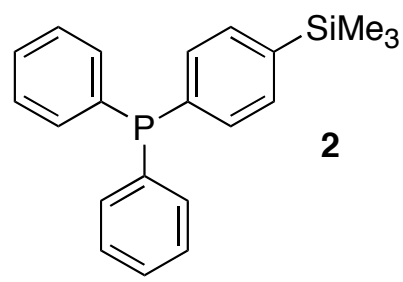

4-(Trimethylsilyl)phenyldiphenylphosphine (2). The title compound was prepared according to the procedure described by van Koten. ${ }^{11}$ A solution of 1-bromo-4-(trimethylsilyl)benzene (3.44 g, $15.0 \mathrm{mmol})$ in diethyl ether $(10 \mathrm{~mL})$ was added to a suspension of magnesium turning in diethyl ether $(10 \mathrm{~mL})$. This solution of the Grignard reagent was then added to a solution of chlorodiphenylphosphine $(3.30 \mathrm{~g}, 15.0 \mathrm{mmol})$ in diethyl ether $(150 \mathrm{~mL})$ at $-78{ }^{\circ} \mathrm{C}$. The cooling

\footnotetext{
${ }^{7}$ Modified procedure according to : Shioiri, T.; Aoyama, T.; Mori, S. Org. Synth. 1990, 68, 1-7.

8 In contrast to diazomethane, trimethylsilyldiazomethane has been shown to be non-explosive and non-mutagenic, thus the very careful operations used for the preparation of diazomethane are not necessary. See reference 7.

${ }^{9}$ Shioiri, T.; Yamada, S. Org. Synth. 1984, 62, 187-190.

${ }^{10}$ Although no risk is associated with the distillation of trimethylsilyldiazomethane (see reference 7), the presence of peroxide from the ether should be considered as a potential danger. The purity of the solution and the presence of peroxide from ether should be always checked prior to perform the distillation.

${ }^{11}$ Richter, B.; de Wolf, E.; van Koten, G.; Deelman, B. J. J. Org. Chem. 2000, 65, 3885-3893.
} 
bath was removed after 2 hours and the reaction mixture was stirred for another 2 hours. When at room temperature, the mixture was quenched with saturated aqueous $\mathrm{NH}_{4} \mathrm{Cl}(25 \mathrm{~mL})$. The two layers were separated and the aqueous layer was further extracted with diethyl ether $(3 \mathrm{x} 50 \mathrm{~mL})$. The combined organic fractions were then dried over $\mathrm{MgSO}_{4}$ and the solvent was removed under reduced pressure. The desired phosphine $2(2.70 \mathrm{~g}, 54 \%)$ was obtained as a white solid after flash chromatography (5\% EtOAc/hexanes). $\mathrm{R}_{f} 0.47$ (5\% EtOAc/hexanes); ${ }^{1} \mathrm{H}$ NMR (400 MHz, $\mathrm{CDCl}_{3}$ ) $\square 7.36-7.35(\mathrm{~m}, 14 \mathrm{H}), 0.29(\mathrm{~s}, 9 \mathrm{H}) ;{ }^{13} \mathrm{C} \mathrm{NMR}\left(100 \mathrm{MHz}, \mathrm{CDCl}_{3}\right) \square 140.9,137.6(\mathrm{~d}, J=11 \mathrm{~Hz})$, $137.0(\mathrm{~d}, J=10 \mathrm{~Hz}), 133.7(\mathrm{~d}, J=20 \mathrm{~Hz}), 133.2(\mathrm{~d}, J=7 \mathrm{~Hz}), 132.7(\mathrm{~d}, J=19 \mathrm{~Hz}), 128.6,128.4$ $(\mathrm{d}, J=7 \mathrm{~Hz}),-1.30 ;{ }^{31} \mathrm{P}$ NMR $\left(162 \mathrm{MHz}, \mathrm{CDCl}_{3}\right) \square-5.07$; IR (neat) 3052, 2953, 1583, 1479, 1435 , 1250, 1122, 839, 744, 696, $631 \mathrm{~cm}^{-1}$; HMRS (MAB) calcd for $\mathrm{C}_{21} \mathrm{H}_{23} \mathrm{PSi}\left[\mathrm{M}-\mathrm{H}^{+}\right]^{+}$: 334.130668 . Found: 334.130051.

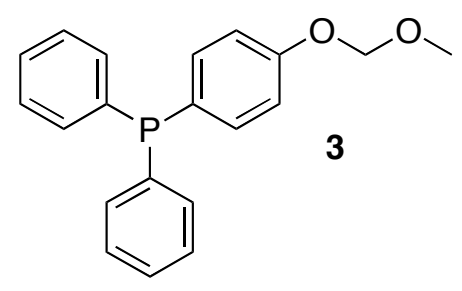

4-(Methoxymethoxy)phenyldiphenylphosphine (3). The title compound was prepared according to the procedure described by Janda. ${ }^{12}$ A solution of $n$-butyl lithium in hexanes $(2.70 \mathrm{~mL}, 6.80$ mmol) was added dropwise, to a stirred solution of 1-bromo-4-methoxymethoxybenzene $(0.74 \mathrm{~g}$, $3.40 \mathrm{mmol})$ in ether $(25 \mathrm{~mL})$ at $0{ }^{\circ} \mathrm{C}$ over $15 \mathrm{~min}$. The resulting mixture was warmed to room temperature and stirred for $3 \mathrm{~h}$, then cooled to $0{ }^{\circ} \mathrm{C}$. Chlorodiphenylphosphine $(0.73 \mathrm{~g}, 3.50 \mathrm{mmol})$ was then added dropwise and the resulting mixture was stirred at room temperature for 16 hours. The reaction was quenched by careful addition of saturated aqueous $\mathrm{NH}_{4} \mathrm{Cl}(50 \mathrm{~mL})$ to the reaction mixture at $0{ }^{\circ} \mathrm{C}$. The two layers were separated and the aqueous layer was further extracted with diethyl ether $(3 \times 50 \mathrm{~mL})$. The combined organic fractions were then dried over $\mathrm{MgSO}_{4}$, and the solvent was removed under reduced pressure. The desired phosphine $3(0.45 \mathrm{~g}, 41 \%)$ was obtained as a white solid after flash chromatography (5\% EtOAc/hexanes). $\mathrm{R}_{f} 0.24$ (5\% EtOAc/hexanes); ${ }^{1} \mathrm{H}$ $\operatorname{NMR}\left(400 \mathrm{MHz}, \mathrm{CDCl}_{3}\right) \square 7.35-7.31(\mathrm{~m}, 10 \mathrm{H}), 7.02-7.07(\mathrm{~m}, J=4 \mathrm{H}), 5.21(\mathrm{~s}, 2 \mathrm{H}), 3.50(\mathrm{~s}, 3 \mathrm{H})$; ${ }^{13} \mathrm{C}$ NMR $\left(100 \mathrm{MHz}, \mathrm{CDCl}_{3}\right) \square 157.9,137.6(\mathrm{~d}, J=11 \mathrm{~Hz}), 135.4(\mathrm{~d}, J=21 \mathrm{~Hz}), 133.4(\mathrm{~d}, J=19$ $\mathrm{Hz}), 129.1(\mathrm{~d}, J=46 \mathrm{~Hz}), 128.9,128.3(\mathrm{~d}, J=7 \mathrm{~Hz}), 116.2(\mathrm{~d}, J=8 \mathrm{~Hz}), 94.1,56.0 ;{ }^{31} \mathrm{P}$ NMR $(162$ $\mathrm{MHz}, \mathrm{CDCl}_{3}$ ) $\square-6.45$; IR (neat) 3052, 2895, 1593, 1496, 1434, 1236, 1152, 1079, 997, 744, 697, $631 \mathrm{~cm}^{-1}$; HMRS (MAB) calcd for $\mathrm{C}_{20} \mathrm{H}_{19} \mathrm{O}_{2} \mathrm{P}\left[\mathrm{M}-\mathrm{H}^{+}\right]^{+}$: 322.112268. Found: 322.111786.<smiles>COC(=O)c1ccc(P(c2ccccc2)c2ccccc2)cc1</smiles>

4-(Diphenylphosphanyl)benzoic acid methyl ester (4). ${ }^{13}$ The title compound was prepared according to the procedure described by Yoakim. ${ }^{14}$ To a solution of 4-(diphenylphosphino)benzoic

12 Sieber, F.; Wentworth, P.; Toker, J. D.; Wentworth, A. D.; Metz, W. A.; Reed, N. N.; Janda, K. D. J. Org. Chem. 1999, 64, 5188-5192.

13 Kwong, F. Y.; Lai, C. W.; Chan, K. S. Tetrahedron Lett. 2002, 43, 3537-3539.

14 Yoakim, C.; Guse, I.; O'Meara, J. A.; Thavonekham, B. Synlett 2003, 473-476. 
acid $(398 \mathrm{mg}, 1.30 \mathrm{mmol})$ in dichloromethane $(15 \mathrm{~mL})$ at $0{ }^{\circ} \mathrm{C}$, was added methanol $(0.05 \mathrm{~mL}, 1.40$ $\mathrm{mmol}$ ) and $N, N$-dimethylaminopyridine $(37.0 \mathrm{mg}, 0.30 \mathrm{mmol})$. A solution of diisopropylcarbodiimide in dichloromethane $(1.40 \mathrm{~mL}, 1.40 \mathrm{mmol})$ was then added over $15 \mathrm{~min}$. The resulting light yellow suspension was stirred at room temperature for 16 hours. The reaction mixture was filtered and concentrated to dryness. The filtrate was diluted with EtOAc $(30 \mathrm{~mL})$, washed successively with aqueous $10 \% \mathrm{HCl}(15 \mathrm{~mL})$, aqueous saturated $\mathrm{NaHCO}_{3}(15 \mathrm{~mL})$ and brine $(15 \mathrm{~mL})$. The organic layer was dried over $\mathrm{MgSO}_{4}$, filtered and evaporated to dryness. The desired phosphine 4 (325 mg, 78\%) was obtained as a white solid after flash chromatography (5\% EtOAc/hexanes). $\mathrm{R}_{f}$ 0.4 (5\% EtOAc/hexanes); ${ }^{1} \mathrm{H}$ NMR $\left(400 \mathrm{MHz}, \mathrm{CDCl}_{3}\right) \square 7.97(\mathrm{~d}, J=7 \mathrm{~Hz}, 2 \mathrm{H}), 7.32-7.38$ (m, $12 \mathrm{H}), 3.91(\mathrm{~s}, 3 \mathrm{H}) ;{ }^{13} \mathrm{C} \mathrm{NMR}\left(100 \mathrm{MHz}, \mathrm{CDCl}_{3}\right) \square 166.7,143.9(\mathrm{~d}, J=14 \mathrm{~Hz}), 136.0(\mathrm{~d}, J=11$ $\mathrm{Hz}), 133.8(\mathrm{~d}, J=20 \mathrm{~Hz}), 133.0(\mathrm{~d}, J=19 \mathrm{~Hz}), 129.9,129.1(\mathrm{~d}, J=7 \mathrm{~Hz}), 129.0,128.6(\mathrm{~d}, J=7$ $\mathrm{Hz}), 52.0 ;{ }^{31} \mathrm{P} \mathrm{NMR}\left(162 \mathrm{MHz}, \mathrm{CDCl}_{3}\right) \square-4.65$.

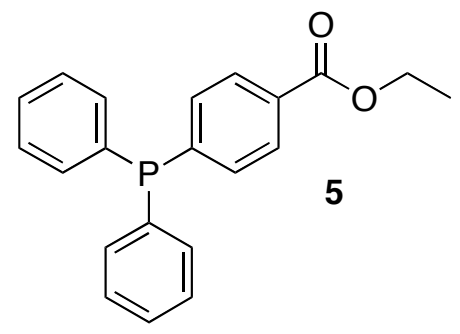

4-(Diphenylphosphanyl)benzoic acid ethyl ester (5). The title compound was prepared from ethanol $(0.07 \mathrm{~mL}, 1.4 \mathrm{mmol})$ according to the procedure described for the synthesis of phosphine 4 . The desired phosphine $5(0.312 \mathrm{mg}, 72 \%)$ was obtained as a white solid after flash chromatography (5\% EtOAc/hexanes). $\mathrm{R}_{f} 0.45$ (5\% EtOAc/hexanes); ${ }^{1} \mathrm{H}$ NMR (400 MHz, $\left.\mathrm{CDCl}_{3}\right) \square 7.99$ (d, $J=8$ $\mathrm{Hz}, 2 \mathrm{H}), 7.32-7.38(\mathrm{~m}, 12 \mathrm{H}), 4.38(\mathrm{q}, J=7 \mathrm{~Hz}, 2 \mathrm{H}), 1.39(\mathrm{t}, J=7 \mathrm{~Hz}, 3 \mathrm{H}) ;{ }^{13} \mathrm{C}$ NMR $(100 \mathrm{MHz}$, $\left.\mathrm{CDCl}_{3}\right) \square 166.3,143.7(\mathrm{~d}, J=14 \mathrm{~Hz}), 136.1(\mathrm{~d}, J=11 \mathrm{~Hz}), 133.8(\mathrm{~d}, J=20 \mathrm{~Hz}), 133.0(\mathrm{~d}, J=19$ $\mathrm{Hz}), 130.2,129.1$ (d, $J=7 \mathrm{~Hz}), 129.0,128.5$ (d, $J=7 \mathrm{~Hz}), 60.9,14.2 ;{ }^{31} \mathrm{P}$ NMR $\left(162 \mathrm{MHz}, \mathrm{CDCl}_{3}\right)$ -4.70; IR (neat) 3053, 2980, 1716, 1597, 1435, 1272, 1108, 1086, 1019, 744, $696 \mathrm{~cm}^{-1}$; HMRS (MAB) calcd for $\mathrm{C}_{21} \mathrm{H}_{19} \mathrm{O}_{2} \mathrm{P}\left[\mathrm{M}-\mathrm{H}^{+}\right]^{+}: 334.112268$. Found: 334.111978.

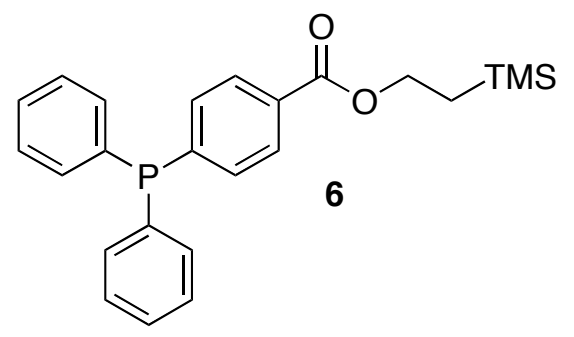

4-(Diphenylphosphanyl)benzoic acid 2-trimethylsilanylethyl ester (DPPBE) (6). ${ }^{10}$ The title compound was prepared from 2-(trimethylsilyl)ethanol $(0.50 \mathrm{~mL}, 3.80 \mathrm{mmol})$ according to the procedure described for the synthesis of phosphine 4. The desired phosphine 6 (1.16 g, 82\%) was obtained as a colorless viscous oil after flash chromatography (5\% EtOAc/hexanes). The reagent was used as a THF solution $(0.5 \mathrm{M})$. ${ }^{1} \mathrm{H}$ NMR $\left(400 \mathrm{MHz}, \mathrm{CDCl}_{3}\right) \square 7.99(\mathrm{~d}, J=7 \mathrm{~Hz}, 2 \mathrm{H}), 7.32-$ $7.38(\mathrm{~m}, 12 \mathrm{H}), 4.44(\mathrm{t}, J=8 \mathrm{~Hz}, 2 \mathrm{H}), 1.14(\mathrm{t}, J=8 \mathrm{~Hz}, 2 \mathrm{H}), 0.10(\mathrm{~s}, 9 \mathrm{H}) ;{ }^{13} \mathrm{C} \mathrm{NMR}(100 \mathrm{MHz}$, $\left.\mathrm{CDCl}_{3}\right) \square 166.4,143.6(\mathrm{~d}, J=14 \mathrm{~Hz}), 136.1(\mathrm{~d}, J=11 \mathrm{~Hz}), 133.8(\mathrm{~d}, J=20 \mathrm{~Hz}), 133.0(\mathrm{~d}, J=22$ $\mathrm{Hz}), 130.4,129.1(\mathrm{~d}, J=6 \mathrm{~Hz}), 129.0,128.5(\mathrm{~d}, J=7 \mathrm{~Hz}), 63.2,17.3,-1.54 ;{ }^{31} \mathrm{P}$ NMR $(162 \mathrm{MHz}$, $\mathrm{CDCl}_{3}$ ) -4.67 ; IR (neat) 3054, 2952, 2896, 1715, 1597, 1435, 1270, 1250, 1108, 1085, 838, 696, $631 \mathrm{~cm}^{-1}$; HMRS (MAB) calcd for $\mathrm{C}_{24} \mathrm{H}_{27} \mathrm{O}_{2} \mathrm{PSi}\left[\mathrm{M}-\mathrm{H}^{+}\right]^{+}$: 406.151797. Found: 406.152982. 


\section{General procedure for the methylenation of aldehydes. Method A. Triphenylphosphine.}

To a solution of chlorotris(triphenylphosphine)rhodium $(0.023 \mathrm{~g}, 0.025 \mathrm{mmol})$ and triphenylphosphine $(0.29 \mathrm{~g}, 1.10 \mathrm{mmol})$ in THF $(10 \mathrm{~mL})$, was added 2-propanol $(75.0 \mu \mathrm{L}, 1.00$ mmol) followed by the aldehyde $(1.00 \mathrm{mmol})$. To the resulting red mixture, was then added a solution of trimethylsilyldiazomethane in THF $(0.82 \mathrm{~mL}, 1.40 \mathrm{mmol})$. Gas evolution was observed and the resulting dark orange mixture was stirred at room temperature. When the reaction is completed by GC or TLC analysis, $3 \% \mathrm{H}_{2} \mathrm{O}_{2}(10 \mathrm{~mL})$ was added and the organic layer was extracted with dichloromethane $(3 \times 10 \mathrm{~mL})$. The combined organic layers were washed with brine $(10 \mathrm{~mL})$ and dried over $\mathrm{MgSO}_{4}$. The solvent was removed under reduced pressure and the crude alkene was purified by flash chromatography on silica gel or by distillation. It is also possible to evaporate directly the reaction mixture and performed a flash chromatography with a pre-absorption on silica.

\section{General procedure for the methylenation of aldehydes. Method B. DPPBE (6).}

To a solution of chlorotris(triphenylphosphine)rhodium $(0.023 \mathrm{~g}, 0.025 \mathrm{mmol})$ in THF $(10 \mathrm{~mL})$ was added a solution of DPPBE (6) in THF $(2.20 \mathrm{~mL}, 1.10 \mathrm{mmol})$, followed by 2-propanol $(75.0 \mu \mathrm{L}$, $1.00 \mathrm{mmol})$ and by the aldehyde $(1.00 \mathrm{mmol})$. To the resulting red mixture, was then added a solution of trimethylsilyldiazomethane in THF $(0.82 \mathrm{~mL}, 1.40 \mathrm{mmol})$. Gas evolution was observed and the resulting dark orange mixture was stirred at room temperature until the reaction is completed by GC or TLC analysis. The reaction mixture was cooled to $0{ }^{\circ} \mathrm{C}$, prior to the addition of a solution of TBAF in THF $(5.00 \mathrm{~mL}, 5.00 \mathrm{mmol})$. The resulting mixture was warmed to room temperature and stirred for 12 hours. The mixture was then diluted with dichloromethane $(30 \mathrm{~mL})$, washed with $10 \%$ aqueoux $\mathrm{NaOH}(15 \mathrm{~mL})$ and brine $(15 \mathrm{~mL})$. The organic layer was dried over $\mathrm{MgSO}_{4}$ and the solvent was removed under reduced pressure. The crude alkene was purified by flash chromatography on silica gel or by distillation.

\section{Wittig procedure for the methylenation of aldehydes. Method C. Methyltriphenylphosphonium bromide and NaHMDS.}

To a solution of methyltriphenylphosphonium bromide (393 mg, $1.10 \mathrm{mmol})$ in THF (10 mL), was added sodium hexamethyldisilazide $(202 \mathrm{mg}, 1.10 \mathrm{mmol})$. The resulting yellow mixture was stirred for 1 hour at room temperature. The aldehyde $(1.00 \mathrm{mmol})$ was then added and the solution was stirred at room temperature until the reaction is completed by GC analysis or by TLC. The solvent was removed under reduced pressure and the crude alkene was purified by flash chromatography on silica gel or by distillation.
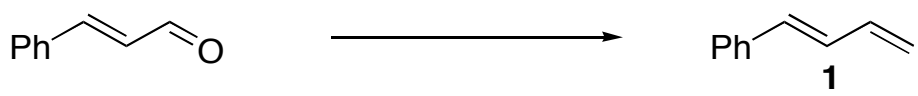

4-Phenyl-1,3-butadiene (1). The title compound was prepared from trans-cinnamaldehyde (250 $u \mathrm{~L}, 2.00 \mathrm{mmol}$ ) according to the general procedure $\mathbf{A}$ (reaction time: $0.5 \mathrm{~h}$ ). The desired alkene $\mathbf{1}$ (230 mg, 88\%) was obtained as a colorless oil after flash chromatography (1\% ether/pentane). $\mathrm{R}_{f}$ 0.63 (1\% EtOAc/hexanes); ${ }^{1} \mathrm{H}$ NMR (400 MHz, $\left.\mathrm{CDCl}_{3}\right) \square 7.43-7.41(\mathrm{~m}, 2 \mathrm{H}), 7.35-7.31$ (m, 2H), 7.26-7.22 (m, 1H), $6.80(\mathrm{dd}, J=16,10 \mathrm{~Hz}, 1 \mathrm{H}), 6.58(\mathrm{~d}, J=16 \mathrm{~Hz}, 1 \mathrm{H}), 6.52(\mathrm{dd}, J=17,10 \mathrm{~Hz}$, $1 \mathrm{H}), 5.35(\mathrm{~d}, J=17 \mathrm{~Hz}, 1 \mathrm{H}), 5.19(\mathrm{~d}, J=10 \mathrm{~Hz}, 1 \mathrm{H})) ;{ }^{13} \mathrm{C} \mathrm{NMR}\left(100 \mathrm{MHz}, \mathrm{CDCl}_{3}\right) \square 137.0$, 136.96, 132.7, 129.5, 128.5, 127.5, 126.3, 117.5; IR (NaCl, film) 3080, 3060, 3030, 1800, 1630, 
1600, 1495, 1450, 1000, 950, 900, 755, $690 \mathrm{~cm}^{-1}$; Anal. Calcd for $\mathrm{C}_{10} \mathrm{H}_{10}: \mathrm{C}, 92.26 ; \mathrm{H}, 7.74$. Found: C, 92.20; H, 8.04.

4-Phenyl-1,3-butadiene (1). The title compound was prepared from trans-cinnamaldehyde (132 $\mathrm{mg}, 1.00 \mathrm{mmol}$ ) according to the general procedure $\mathbf{B}$ (reaction time: $2 \mathrm{~h}$ ). The desired alkene $\mathbf{1}$ (107 mg, 82\%) was obtained as a colorless oil after flash chromatography (1\% ether/pentane).

4-Phenyl-1,3-butadiene (1). The title compound was prepared from trans-cinnamaldehyde (132 $\mathrm{mg}, 1.00 \mathrm{mmol}$ ) according to the general procedure $\mathbf{C}$ (reaction time: $2 \mathrm{~h}$ ). The desired alkene $\mathbf{1}$ (107 mg, 82\%) was obtained as a colorless oil after flash chromatography (1\% ether/pentane).

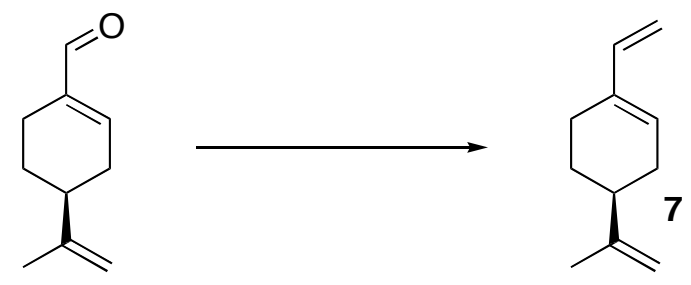

(S)-4-Isopropenyl-1-vinyl-cyclohexene (7). The title compound was prepared from (S)-(-)perillaldehyde $(750 \mathrm{mg}, 5.00 \mathrm{mmol}$ ) according to the general procedure $\mathbf{B}$ (reaction time: $5 \mathrm{~h}$ ). The desired alkene 7 (585 mg, 79\%) was obtained as a colorless oil after kugelrhor distillation (b.p. 95 $\left.{ }^{\circ} \mathrm{C}\right) . \quad \mathrm{R}_{f} 0.60$ (1\% EtOAc/hexanes); ${ }^{1} \mathrm{H}$ NMR $\left(400 \mathrm{MHz}, \mathrm{CDCl}_{3}\right) \square 6.38(\mathrm{dd}, J=17,11 \mathrm{~Hz}, 1 \mathrm{H})$, $5.78(\mathrm{~m}, 1 \mathrm{H}), 5.08(\mathrm{~d}, J=17 \mathrm{~Hz}, 1 \mathrm{H}), 4.92(\mathrm{~d}, J=11 \mathrm{~Hz}, 1 \mathrm{H}), 4.75(\mathrm{~s}, 1 \mathrm{H}), 4.74(\mathrm{~s}, 1 \mathrm{H}), 2.36-2.07$ (m, 6H), 1.94-1.89 (m, 1H), $1.76(\mathrm{~s}, 1 \mathrm{H}) ;{ }^{13} \mathrm{C} \mathrm{NMR}\left(100 \mathrm{MHz}, \mathrm{CDCl}_{3}\right) \square$ 149.6, 139.5, 135.6, 129.0, 109.9, 108.6, 41.1, 31.1, 27.2, 24.1, 20.6; IR (neat) 3066, 3086, 2920, 1645, 1436, 989, 890, 835 $\mathrm{cm}^{-1}$; HMRS (MAB) calcd for $\mathrm{C}_{11} \mathrm{H}_{16}[\mathrm{M}]^{+}:$148.125201. Found: 148.125212.

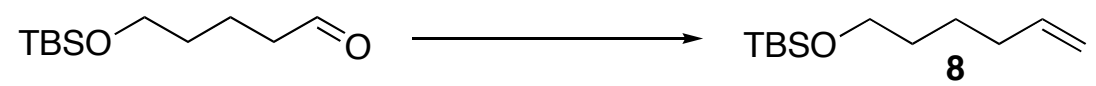

6-(tert-Butyldimethylsilyloxy)-1-hexene (8). The title compound was prepared from 6-(tertbutyldimethylsilyloxy)-1-pentanal $(216 \mathrm{mg}, 1.0 \mathrm{mmol})$ according to the general procedure A (reaction time: $1 \mathrm{~h}$ ). The desired alkene $\mathbf{8}(186 \mathrm{mg}, 87 \%)$ was obtained as a colorless oil after flash chromatography (2\% EtOAc/hexanes). $\mathrm{R}_{f} 0.48$ (2\% EtOAc/hexanes); ${ }^{1} \mathrm{H} \mathrm{NMR}\left(400 \mathrm{MHz}, \mathrm{CDCl}_{3}\right)$ $\square$ 5.84-5.78 (m, 1H), $5.00(\mathrm{~d}, J=17 \mathrm{~Hz}, 1 \mathrm{H}), 4.94(\mathrm{~d}, J=11 \mathrm{~Hz}, 1 \mathrm{H}), 3.61(\mathrm{t}, J=6 \mathrm{~Hz}, 2 \mathrm{H}), 2.09$ $2.04(\mathrm{~m}, 2 \mathrm{H}), 1.55-1.50(\mathrm{~m}, 2 \mathrm{H}), 1.47-1.41(\mathrm{~m}, 2 \mathrm{H}), 0.90(\mathrm{~s}, 9 \mathrm{H}), 0.05(\mathrm{~s}, 6 \mathrm{H}) ;{ }^{13} \mathrm{C} \mathrm{NMR}(100$ $\left.\mathrm{MHz}, \mathrm{CDCl}_{3}\right) \square 138.8,114.2,62.9,33.4,32.1,25.8,25.0,18.2$; IR (NaCl, film) 3080, 2935 (br), $1640,1470,1390,1360,1255,1110,910,840,775,660 \mathrm{~cm}^{-1}$; Anal. Calcd for $\mathrm{C}_{12} \mathrm{H}_{26} \mathrm{OSi}$ : C, 67.22; H, 12.22. Found: C, 65.94; H, 12.41.

6-(tert-Butyldimethylsilyloxy)-1-hexene (8). The title compound was prepared from 6-(tertbutyldimethylsilyloxy)-1-pentanal $(216 \mathrm{mg}, 1.00 \mathrm{mmol})$ according to the general procedure $\mathbf{C}$ (reaction time: $7 \mathrm{~h}$ ). The desired alkene $\mathbf{8}(152 \mathrm{mg}, 71 \%)$ was obtained as a colorless oil after flash chromatography (2\% EtOAc/hexanes).
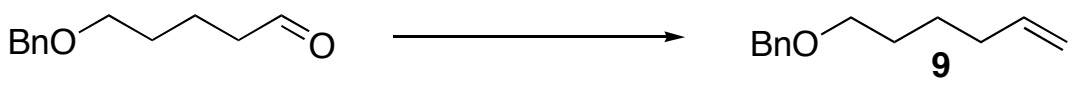
6-(Benzyloxy)-1-hexene (9). The title compound was prepared from 5-benzyloxy-1-pentanal15 (192 $\mathrm{mg}, 1.00 \mathrm{mmol}$ ) according to the general procedure $\mathbf{A}$ (reaction time: $0.5 \mathrm{~h}$ ). The desired alkene 9 (141 mg, 74\%) was obtained as a colorless oil after flash chromatography (1\% EtOAc/hexanes). $\mathrm{R}_{f}$ 0.42 (1\% EtOAc/hexanes); ${ }^{1} \mathrm{H}$ NMR $\left(300 \mathrm{MHz}, \mathrm{CDCl}_{3}\right) \square 7.37-7.26(\mathrm{~m}, 5 \mathrm{H}), 5.90-5.76(\mathrm{~m}, 1 \mathrm{H})$, 5.06-4.94 (m, 2H), $4.52(\mathrm{~s}, 2 \mathrm{H}), 3.50(\mathrm{t}, J=6 \mathrm{~Hz}, 2 \mathrm{H}), 2.13-2.05(\mathrm{~m}, 2 \mathrm{H}), 1.69-1.62(\mathrm{~m}, 2 \mathrm{H}), 1.55-$ $1.47(\mathrm{~m}, 2 \mathrm{H}) ;{ }^{13} \mathrm{C}$ NMR $\left(75 \mathrm{MHz}, \mathrm{CDCl}_{3}\right) \square 139.2,139.1,128.8,128.0,127.9,114.9,73.3,70.7$, 34.0, 29.6, 25.9; IR (neat) 3066, 3032, 2933, 2858, 1641, 1455, 1362, 1103, 909, 734, $631 \mathrm{~cm}^{-1}$; HMRS (MAB) calcd for $\mathrm{C}_{13} \mathrm{H}_{18} \mathrm{O}[\mathrm{M}]^{+}:$190.135765. Found: 190.135287.

6-(Benzyloxy)-1-hexene (9). The title compound was prepared from 5-benzyloxy-pentanal ${ }^{11}$ (192 $\mathrm{mg}, 1.00 \mathrm{mmol}$ ) according to the general procedure $\mathbf{B}$ (reaction time: $0.5 \mathrm{~h}$ ). The desired alkene $\mathbf{9}$ (169 mg, 89\%) was obtained as a colorless oil after flash chromatography (1\% EtOAc/hexanes).

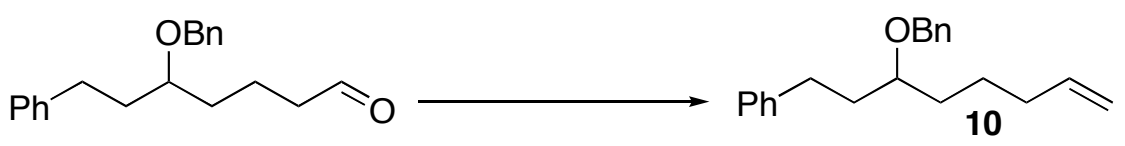

7-Benzyloxy-4-phenyl-1-octene (10). The title compound was prepared from 7-benzyloxy-4phenyl-1-heptanal $(583 \mathrm{mg}, 1.97 \mathrm{mmol})$ according to the general procedure A (reaction time: $1 \mathrm{~h})$. The desired alkene $\mathbf{1 0}(570 \mathrm{mg}, 98 \%)$ was obtained as a colorless oil after flash chromatography (2\% EtOAc/hexanes). $\mathrm{R}_{f} 0.70$ (2\% EtOAc/hexanes); ${ }^{1} \mathrm{H}$ NMR (400 MHz, $\left.\mathrm{CDCl}_{3}\right) \square 7.39-7.26$ (m, $6 \mathrm{H}), 7.21-7.18(\mathrm{~m}, 4 \mathrm{H}), 5.87-5.77(\mathrm{~m}, 1 \mathrm{H}), 5.05-4.95(\mathrm{~m}, 2 \mathrm{H}), 4.54(\mathrm{~d}, J=12 \mathrm{~Hz}, 1 \mathrm{H}), 4.51(\mathrm{~d}, J=$ $12 \mathrm{~Hz}, 1 \mathrm{H}), 3.47-3.41(\mathrm{~m}, 1 \mathrm{H}), 2.81-2.73(\mathrm{~m}, 1 \mathrm{H}), 2.70-2.63(\mathrm{~m}, 1 \mathrm{H}), 2.10-2.04(\mathrm{~m}, 2 \mathrm{H}), 1.94-1.80$ $(\mathrm{m}, 2 \mathrm{H}), 1.68-1.44(\mathrm{~m}, 4 \mathrm{H}) ;{ }^{13} \mathrm{C}$ NMR $\left(100 \mathrm{MHz}, \mathrm{CDCl}_{3}\right) \square 142.3,138.8,138.6,128.3,128.2(2)$, 127.7, 127.4, 125.6, 114.4, 78.0, 70.6, 35.5, 33.7, 33.0, 31.5, 24.4; IR (NaCl, film) 3065, 3030, 2935, 2860, 1640, 1495, 1455, 1360, 1095, 1070, 910, 840, 735, $700 \mathrm{~cm}^{-1}$; Anal. Calcd for $\mathrm{C}_{21} \mathrm{H}_{26} \mathrm{O}: \mathrm{C}, 85.67 ; \mathrm{H}, 8.90$. Found: C, 85.09; H, 8.95.

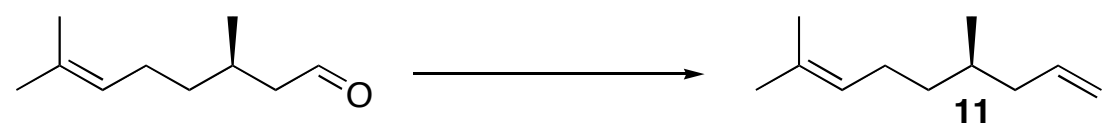

(R)-2,6-Dimethyl-2,8-nonadiene (11). The title compound was prepared from $(R)$-citronellal (360 $u \mathrm{~L}, 2.00 \mathrm{mmol}$ ) according to the general procedure $\mathbf{A}$ (reaction time: $7 \mathrm{~h}$ ). The desired alkene $\mathbf{1 1}$ (256 mg, 84\%) was obtained as a colorless oil after flash chromatography (2\% EtOAc/hexanes). $\mathrm{R}_{f}$ 0.48 (2\% EtOAc/hexanes); ${ }^{1} \mathrm{H}$ NMR $\left(400 \mathrm{MHz}, \mathrm{CDCl}_{3}\right) \square 5.84-5.74(\mathrm{~m}, 1 \mathrm{H}), 5.13-5.09(\mathrm{~m}, 1 \mathrm{H})$, 5.03-4.96 (m, 2H), 2.13-1.86 (m, 4H), $1.69(\mathrm{~s}, 3 \mathrm{H}), 1.61(\mathrm{~s}, 3 \mathrm{H}), 1.57-1.47(\mathrm{~m}, 1 \mathrm{H}), 1.40-1.30(\mathrm{~m}$, $1 \mathrm{H}), 1.20-1.11(\mathrm{~m}, 1 \mathrm{H}), 0.89(\mathrm{~d}, J=7 \mathrm{~Hz}, 6 \mathrm{H}) ;{ }^{13} \mathrm{C} \mathrm{NMR}\left(100 \mathrm{MHz}, \mathrm{CDCl}_{3}\right) \square 137.5,131.0,124.7$, 115.3, 41.2, 36.5, 32.2, 25.6, 25.4, 19.2, 17.5; IR (NaCl, film) 3080, 2965, 2915, 1640, 1455, 1380, 995, $910 \mathrm{~cm}^{-1}$; Anal. Calcd for $\mathrm{C}_{11} \mathrm{H}_{20}: \mathrm{C}, 86.76 ; \mathrm{H}, 13.24$. Found: C, 86.81; H, 13.23.

$(\boldsymbol{R})$-2,6-Dimethyl-2,8-nonadiene (11). The title compound was prepared from $(R)$-citronellal (180 $u \mathrm{~L}, 1.00 \mathrm{mmol}$ ) according to the general procedure $\mathbf{B}$ (reaction time: $5 \mathrm{~h}$ ). The desired alkene $\mathbf{1 1}$ (131 mg, 86\%) was obtained as a colorless oil after flash chromatography (2\% EtOAc/hexanes).

15 Crimmins, M. T.; Katz, J. D.; McAtee, L. C.; Tabet, E. A.; Kirincich, S. J. Org. Lett. 2001, 3, 949-952. 


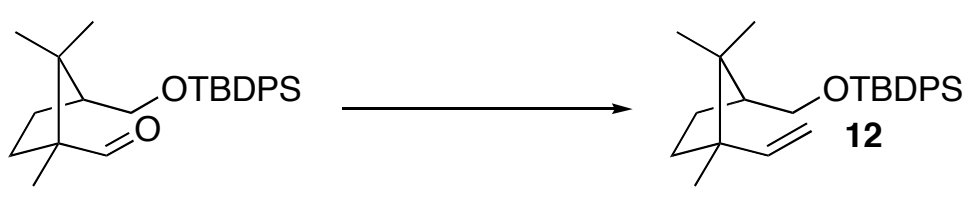

1-[3-(tert-Butyldiphenylsilyloxymethyl)-1,2,2-trimethylcyclopentyl]ethane (12). The title compound was prepared from 3-(tert-butyl-diphenyl-silanyloxymethyl)-1,2,2-trimethyl-cyclopentanecarbaldehyde ${ }^{16}(410 \mathrm{mg}, 1.00 \mathrm{mmol})$ according to the general procedure $\mathbf{A}$ (reaction time: 7 h). The desired alkene 12 (320 mg, 79\%) was obtained as a colorless oil after flash chromatography (5\% EtOAc/hexanes). $\mathrm{R}_{f} 0.57$ (5\% EtOAc/hexanes); ${ }^{1} \mathrm{H}$ NMR $\left(400 \mathrm{MHz}, \mathrm{CDCl}_{3}\right) \square 7.70-7.67$ (m, 4H), 7.43-7.37 (m, 6H), $5.87(\mathrm{dd}, J=17,11 \mathrm{~Hz}, 1 \mathrm{H}), 4.97(\mathrm{~d}, J=11 \mathrm{~Hz}, 1 \mathrm{H}), 4.91(\mathrm{~d}, J=17 \mathrm{~Hz}$, $1 \mathrm{H}), 3.71(\mathrm{dd}, J=10,7 \mathrm{~Hz}, 1 \mathrm{H}), 3.56(\mathrm{dd}, J=10,7 \mathrm{~Hz}, 1 \mathrm{H}), 2.22-2.14(\mathrm{~m}, 1 \mathrm{H}), 1.94-1.82(\mathrm{~m}, 2 \mathrm{H})$, 1.40-1.25 (m, 2H), $1.06(\mathrm{~s}, 9 \mathrm{H}), 0.95(\mathrm{~s}, 3 \mathrm{H}), 0.90(\mathrm{~s}, 3 \mathrm{H}), 0.66(\mathrm{~s}, 3 \mathrm{H}) ;{ }^{13} \mathrm{C}$ NMR $(100 \mathrm{MHz}$, $\left.\mathrm{CDCl}_{3}\right) \square$ 144.9, 135.5, 133.9, 129.4, 127.4, 111.7, 65.8, 50.9, 48.8, 44.5, 34.3, 26.7, 24.9, 22.6, 22.2, 19.4, 19.1; IR (NaCl, film) 3070, 2960, 2860, 1470, 1430, 1110, 1070, 825, $700 \mathrm{~cm}^{-1}$; Anal. Calcd for $\mathrm{C}_{27} \mathrm{H}_{38} \mathrm{OSi}: \mathrm{C}, 79.74 ; \mathrm{H}, 9.42$. Found: C, 78.35; H, 9.16. HMRS (FAB) calcd for $\mathrm{C}_{27} \mathrm{H}_{39} \mathrm{OSi}\left[\mathrm{M}^{+} \mathrm{H}\right]^{+}:$407.27701. Found: 407.27790.
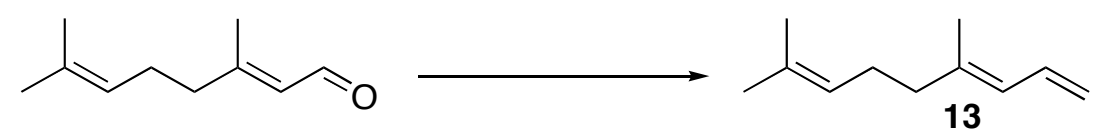

(E)-4,8-Dimethyl-nona-1,3,7-triene (13). ${ }^{17}$ The title compound was prepared from citral (152 mg, $1.00 \mathrm{mmol}$ ) according to the general procedure $\mathbf{A}$ (reaction time: $2 \mathrm{~h}$ ). The desired alkene $\mathbf{1 3}$ (135 $\mathrm{mg}, 90 \%)$ was obtained as a colorless oil after flash chromatography $(1 \%$ EtOAc/hexanes $) . \mathrm{R}_{f} 0.68$ (1\% EtOAc/hexanes); ${ }^{1} \mathrm{H}$ NMR $\left(400 \mathrm{MHz}, \mathrm{CDCl}_{3}\right) \square 6.59$ (dt, $\left.J=17,10 \mathrm{~Hz}, 1 \mathrm{H}\right), 5.88(\mathrm{~d}, J=10$ $\mathrm{Hz}, 1 \mathrm{H}), 5.14-5.08(\mathrm{~m}, 2 \mathrm{H}), 5.00(\mathrm{~d}, J=10 \mathrm{~Hz}, 1 \mathrm{H}), 2.20-2.08(\mathrm{~m}, 4 \mathrm{H}), 1.78(\mathrm{~s}, 3 \mathrm{H}), 1.70(\mathrm{~s}, 3 \mathrm{H})$, $1.62(\mathrm{~s}, 3 \mathrm{H}) ;{ }^{13} \mathrm{C}$ NMR $\left(100 \mathrm{MHz}, \mathrm{CDCl}_{3}\right) \square 139.9,133.8,132.1,125.8,124.4,115.0,40.3,26.9$, 26.1, 18.1, 17.1; IR (neat) 3080, 2965, 2915, 1640, 1455, 1380, 995, $910 \mathrm{~cm}^{-1}$.

(E)-4,8-Dimethyl-nona-1,3,7-triene (13). The title compound was prepared from citral (152 mg, $1.00 \mathrm{mmol}$ ) according to the general procedure $\mathbf{B}$ (reaction time: $2 \mathrm{~h}$ ). The desired alkene $\mathbf{1 3}$ (116 $\mathrm{mg}, 77 \%$ ) was obtained as a colorless oil after flash chromatography (1\% EtOAc/hexanes).

(E)-4,8-Dimethyl-nona-1,3,7-triene (13). The title compound was prepared from citral (152 mg, $1.00 \mathrm{mmol}$ ) according to the general procedure $\mathbf{C}$ (reaction time: $6 \mathrm{~h}$ ). The desired alkene $\mathbf{1 3}$ (116 $\mathrm{mg}, 77 \%$ ) was obtained as a colorless oil after flash chromatography (1\% EtOAc/hexanes).

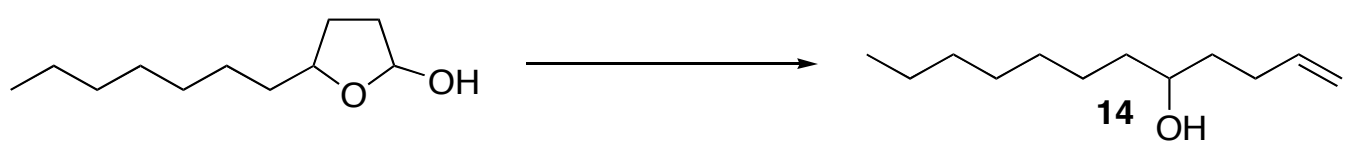

Dodec-1-en-5-ol (14). The title compound was prepared from 5-heptyl-tetrahydro-furan-2-ol ${ }^{18}$ (93 $\mathrm{mg}, 0.50 \mathrm{mmol})$ with 2-methyl-1-propanol $(1 \mathrm{~mL})$ according to the general procedure $\mathbf{A}$ (reaction time: $1 \mathrm{~h})$. The desired alkene $14(58 \mathrm{mg}, 63 \%)$ was obtained as a colorless oil after flash chromatography (5\% EtOAc/hexanes). $\mathrm{R}_{f} 0.22$ (10\% EtOAc/hexanes); ${ }^{1} \mathrm{H}$ NMR (300 MHz, $\left.\mathrm{CDCl}_{3}\right) \square 5.82(\mathrm{ddt}, J=17,10,7 \mathrm{~Hz}, 1 \mathrm{H}), 5.01(\mathrm{~d}, J=17 \mathrm{~Hz}, 1 \mathrm{H}), 4.94(\mathrm{~d}, J=10, \mathrm{~Hz}, 1 \mathrm{H}), 3.63-$

\footnotetext{
16 Betancort, J. M.; Rodriguez, C. M.; Martin, V. S. Tetrahedron Lett. 1998, 39, 9773-9776.

17 Leopold, E. J. Org. Synth. 1986, 64, 164-174.

18 Brunetiere, A. P.; Lallemand, J. Y. Tetrahedron Lett. 1988, 29, 2179-2182.
} 
$3.55(\mathrm{~m}, 1 \mathrm{H}), 2.22-2.04(\mathrm{~m}, 2 \mathrm{H}), 1.60-1.16(\mathrm{~m}, 14 \mathrm{H}), 0.85(\mathrm{t}, J=5 \mathrm{~Hz}, 3 \mathrm{H}) ;{ }^{13} \mathrm{C}$ NMR $(75 \mathrm{MHz}$, $\left.\mathrm{CDCl}_{3}\right) \square 139.1,115.2,72.0,37.9,36.9,32.3,30.6,30.1,29.7,26.1,23.1,14.6$; IR (neat) 3339 (br), 2956, 2924, 2855, 1641, 1456, 1378, 909, $630 \mathrm{~cm}^{-1}$; HMRS (MAB) calcd for $\mathrm{C}_{12} \mathrm{H}_{24} \mathrm{O}[\mathrm{M}]^{+}$: 184.182716. Found: 184.182550.

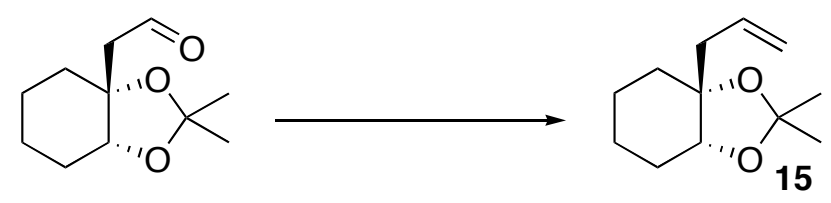

$\left(1 R^{*}, 5 R *\right)-1-A l l y l-3,3-d i m e t h y l-2,4-d i o x a b i c y c l o[4,3,0]$ nonane $(15)$. The title compound was prepared from $\left(1 R^{*}, 2 R^{*}\right)$-[(1,2-isopropylidenedioxy)cyclohexyl]acetaldehyde ${ }^{19}(56.0 \mathrm{mg}, 0.280$ mmol) according to the general procedure $\mathbf{A}$ (reaction time: $1.5 \mathrm{~h}$ ). The desired alkene 15 (43.0 $\mathrm{mg}$, 79\%) was obtained as a colorless oil after flash chromatography (5\% Ether/pentane). $\mathrm{R}_{f} 0.36$ (5\% Ether/pentane); ${ }^{1} \mathrm{H}$ NMR (400 MHz, $\left.\mathrm{CDCl}_{3}\right) \square 5.98-5.88(\mathrm{~m}, 1 \mathrm{H}), 5.13-5.10(\mathrm{~m}, 2 \mathrm{H}), 3.93(\mathrm{~s}, 1 \mathrm{H})$, $2.39(\mathrm{dd}, J=14,7 \mathrm{~Hz}, 1 \mathrm{H}), 2.31(\mathrm{dd}, J=14,7 \mathrm{~Hz}, 1 \mathrm{H}), 2.10-2.07(\mathrm{~m}, 1 \mathrm{H}), 1.71-1.48(\mathrm{~m}, 5 \mathrm{H}), 1.52$ $(\mathrm{s}, 3 \mathrm{H}), 1.35(\mathrm{~s}, 3 \mathrm{H}), 1.21-1.11(\mathrm{~m}, 1 \mathrm{H}), 0.90-0.87(\mathrm{~m}, 1 \mathrm{H}) ;{ }^{13} \mathrm{C} \mathrm{NMR}\left(100 \mathrm{MHz}, \mathrm{CDCl}_{3}\right) \square 134.3$, $118.1,107.2,80.5,76.6,40.5,34.7,28.9,27.4,26.6,23.1,20.2$.

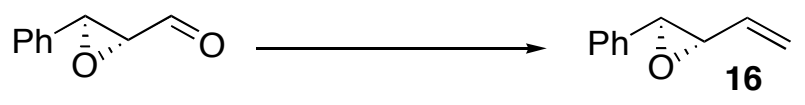

(2S,3S)-2-Phenyl-3-vinyloxirane (16). The title compound was prepared from $(2 R, 3 S)$-3-phenyloxirane-2-carbaldehyde ${ }^{20}(228 \mathrm{mg}, 1.50 \mathrm{mmol})$ according to the general procedure $\mathbf{A}$ (reaction time: $1 \mathrm{~h})$. The desired alkene $16(188 \mathrm{mg}, 86 \%)$ was obtained as a colorless oil after flash chromatography (5\% EtOAc/hexanes). $\mathrm{R}_{f} 0.49$ (5\% EtOAc/hexanes); ${ }^{1} \mathrm{H}$ NMR $\left(400 \mathrm{MHz}, \mathrm{C}_{6} \mathrm{D}_{6}\right) \square$ 7.25-7.15 (m, 5H), 5.65-5.56 (m, 1H), $5.32(\mathrm{~d}, J=17 \mathrm{~Hz}, 1 \mathrm{H}), 5.11(\mathrm{~d}, J=10 \mathrm{~Hz}, 1 \mathrm{H}), 3.53(\mathrm{~s}, 1 \mathrm{H})$, $3.18(\mathrm{~d}, J=7 \mathrm{~Hz}, 2 \mathrm{H}) ;{ }^{13} \mathrm{C}$ NMR $\left(100 \mathrm{MHz}, \mathrm{C}_{6} \mathrm{D}_{6}\right) \square 138.2,136.2,129.0,128.6,126.2,119.1,63.3$, 60.6; IR (NaCl, film) 3080, 3025, 2980, 1495, 1460, 1440, 870, $750 \mathrm{~cm}^{-1}$; HMRS (EI') calcd for $\mathrm{C}_{10} \mathrm{H}_{9} \mathrm{O}\left[\mathrm{M}-\mathrm{H}^{+}\right]^{+}:$145.065340. Found: 145.065174 .
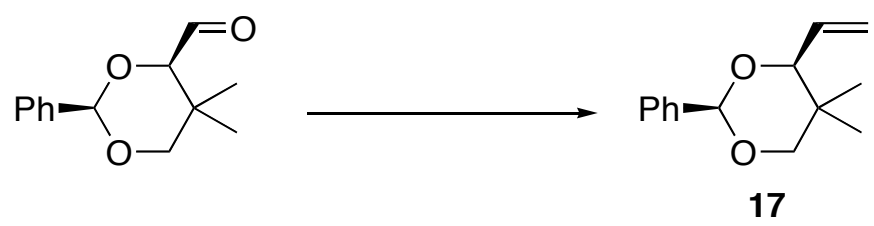

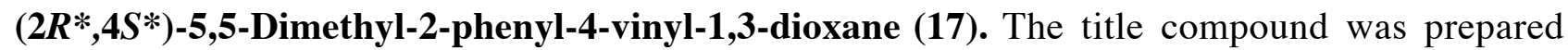
from $\left(2 R^{*}, 4 S^{*}\right)$-4-formyl-5,5-Dimethyl-2-phenyl-1,3-dioxane ${ }^{21}$ (280 $\mathrm{mg}, 1.25 \mathrm{mmol}$ ) according to the general procedure A (reaction time: $5 \mathrm{~h}$ ). The desired alkene 17 (202 $\mathrm{mg}, 74 \%)$ was obtained as a colorless oil after flash chromatography (5\% EtOAc/hexanes). $\mathrm{R}_{f} 0.52(5 \% \mathrm{EtOAc} / \mathrm{hexanes}) ;{ }^{1} \mathrm{H}$ NMR (400 MHz, $\left.\mathrm{C}_{6} \mathrm{D}_{6}\right) \square 7.67-7.64(\mathrm{~m}, 2 \mathrm{H}), 7.18-7.07(\mathrm{~m}, 3 \mathrm{H}), 5.71(\mathrm{ddd}, J=17,10,6 \mathrm{~Hz}, 1 \mathrm{H})$, $5.36(\mathrm{~s}, 1 \mathrm{H}), 5.26(\mathrm{~d}, J=17 \mathrm{~Hz}, 1 \mathrm{H}), 5.04(\mathrm{~d}, J=10 \mathrm{~Hz}, 1 \mathrm{H}), 3.70(\mathrm{~d}, J=7 \mathrm{~Hz}, 1 \mathrm{H}), 3.50(\mathrm{~d}, J=11$ $\mathrm{Hz}, 1 \mathrm{H}), 3.23(\mathrm{~d}, J=11 \mathrm{~Hz}, 1 \mathrm{H}), 1.06(\mathrm{~s}, 3 \mathrm{H}), 0.40(\mathrm{~s}, 3 \mathrm{H}) ;{ }^{13} \mathrm{C} \mathrm{NMR}\left(100 \mathrm{MHz}, \mathrm{C}_{6} \mathrm{D}_{6}\right) \square 136.8$, 131.5, 125.9, 125.4, 123.9, 114.0, 98.9, 82.9, 75.5, 30.1, 18.4, 16.1; IR (NaCl, film) 3070, 2960, $2840,1460,1390,1135,1100,1030,990,750,700 \mathrm{~cm}^{-1}$; HMRS (MAB) calcd for $\mathrm{C}_{14} \mathrm{H}_{17} \mathrm{O}_{2}$ [M$\left.\mathrm{H}^{+}\right]^{+}:$217.122855. Found: 217.121927.

19 Devaux, J. M.; Gore, J.; Vatele, J. M. Tetrahedron: Asymmetry 1998, 9, 1619-1626.

20 Evans, D. A.; Williams, J. M. Tetrahedron Lett. 1988, 29, 5065-5068.

21 Ito, M.; Kibayashi, C. Synthesis 1993, 137-140. 

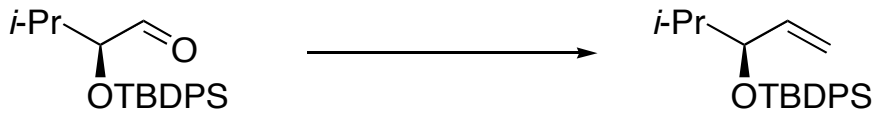

18

(3S)-3-(tert-Butyldiphenylsilyloxy)-4-methyl-1-pentene (18). The title compound was prepared from (2S)-2-(tert-butyldiphenylsilyloxy)-3-methylbutanal $(618 \mathrm{mg}, 1.80 \mathrm{mmol}$; prepared from enantiomerically pure L-leucine ${ }^{22}$ ) according to the general procedure $\mathbf{A}$ (reaction time: $3 \mathrm{~h}$ ). The desired alkene 18 (540 mg, 89\%) was obtained as a colorless oil after flash chromatography (5\% EtOAc/hexanes). $\mathrm{R}_{f} 0.55$ (5\% EtOAc/hexanes); [ $\left.\square\right]_{\mathrm{D}}{ }^{25}=+21.7\left(\mathrm{c} 0.18, \mathrm{CHCl}_{3}\right) ;\left(\right.$ lit. $^{18}[\square]_{\mathrm{D}}{ }^{25}=+22.8$ (c 0.18, $\left.\mathrm{CHCl}_{3}\right)$ ); ${ }^{1} \mathrm{H}$ NMR $\left(400 \mathrm{MHz}, \mathrm{CDCl}_{3}\right) \square 7.69-7.74(\mathrm{~m}, 4 \mathrm{H}), 7.36-7.46(\mathrm{~m}, 6 \mathrm{H}), 5.81(\mathrm{ddd}, J$ $=17,10,7 \mathrm{~Hz}, 1 \mathrm{H}), 5.00(\mathrm{~d}, J=10 \mathrm{~Hz}, 1 \mathrm{H}), 4.93(\mathrm{~d}, J=17 \mathrm{~Hz}, 1 \mathrm{H}), 4.00(\mathrm{dd}, J=7,4 \mathrm{~Hz}, 1 \mathrm{H})$, $1.73-1.78(\mathrm{~m}, 1 \mathrm{H}), 1.12(\mathrm{~s}, 9 \mathrm{H}), 0.89(\mathrm{~d}, J=7 \mathrm{~Hz}, 3 \mathrm{H}), 0.82(\mathrm{~d}, J=7 \mathrm{~Hz}, 3 \mathrm{H}) ;{ }^{13} \mathrm{C} \mathrm{NMR}(100 \mathrm{MHz}$, $\left.\mathrm{CDCl}_{3}\right) \square 137.8,135.9,135.8,134.4,129.3,129.2,127.34,127.27,127.1,115.6,79.5,34.1,27.0$, 19.4, 18.2, 16.8; IR (NaCl, film) 1070, 2960, 2860, 1475, 1430, 1110, 1060, 820, 740, $700 \mathrm{~cm}^{-1}$; HMRS (FAB) calcd for $\mathrm{C}_{22} \mathrm{H}_{29} \mathrm{OSi}\left[\mathrm{M}-\mathrm{H}^{+}\right]^{+}$: 337.19876. Found: 337.19640 .
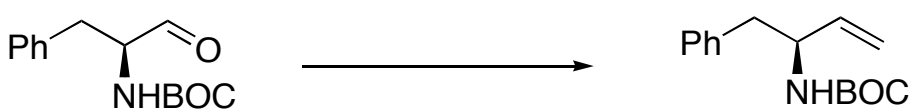

19

(S)-2-(tert-Butoxycarbonylamino)-1-phenylbut-3-ene (19). ${ }^{23}$ The title compound was prepared from $(S)$-2-(tert-butoxycarbonylamino)-3-phenylpropan-1-al (249 mg, $1.00 \mathrm{mmol}$; prepared from enantiomerically pure L-phenylalanine ${ }^{24}$ ) according to the general procedure A (reaction time: 16 h). The desired alkene $\mathbf{1 9}(131 \mathrm{mg}, 53 \%)$ was obtained as a colorless oil after flash chromatography (5\% EtOAc/hexanes). $\mathrm{R}_{f} 0.22\left(5 \%\right.$ EtOAc/hexanes); $[\square]_{\mathrm{D}}{ }^{25}=+5.15$ (c 2.0, $\left.\mathrm{CHCl}_{3}\right) ;{ }^{1} \mathrm{H} \mathrm{NMR}$ $\left(400 \mathrm{MHz}, \mathrm{CDCl}_{3}\right) \square 7.15-7.33(\mathrm{~m}, 5 \mathrm{H}), 5.80(\mathrm{ddd}, J=16,10,5 \mathrm{~Hz}, 1 \mathrm{H}), 5.17(\mathrm{dd}, J=10,1 \mathrm{~Hz}$, $1 \mathrm{H}), 5.12$ (dd, $J=16,1 \mathrm{~Hz}, 1 \mathrm{H}), 4.42$ (s (br), 2H), 2.84 (d (br), $J=7 \mathrm{~Hz}, 2 \mathrm{H}), 1.42(\mathrm{~s}, 9 \mathrm{H}) ;{ }^{13} \mathrm{C}$ NMR (100 MHz, $\left.\mathrm{CDCl}_{3}\right) \square$ 155.6, 138.5, 137.8, 130.0, 128.8, 126.9, 115.2, 79.9, 53.8, 41.9, 28.8; The enantiomeric excess was determined to be $95 \%$ (39:1) following a procedure described in the literature. ${ }^{19}$

(S)-2-(tert-Boc-amino)-1-phenylbut-3-ene (19). The title compound was prepared from $(S)$-2-(tertbutoxycarbonylamino)-3-phenylpropan-1-al (249 mg, $1.00 \mathrm{mmol}$; prepared from enantiomerically pure L-phenylalanine ${ }^{24}$ ) according to the general procedure $\mathbf{B}$ (reaction time $16 \mathrm{~h}$ ). The desired alkene 19 (168 mg, 68\%) was obtained as a colorless oil after flash chromatography (5\% $\mathrm{EtOAc/hexanes).} \mathrm{The} \mathrm{enantiomeric} \mathrm{excess} \mathrm{was} \mathrm{determined} \mathrm{to} \mathrm{be} 95 \%$ (39:1) following a procedure described in the literature. ${ }^{19}$

22 Ina, H.; Ito, M.; Kibayashi, C. J. Org. Chem. 1996, 61, 1023-1029.

23 Luly, J. R.; Dellaria, J. F.; Plattner, J. J.; Soderquist, J. L.; Yi, N. J. Org. Chem. 1987, 52, 1487-1492.

24 Crude aldehyde prepared by TEMPO oxidation, see : Noula, C.; Loukas, V.; Kokotos, G. Synthesis 2002, 1735 1739. 

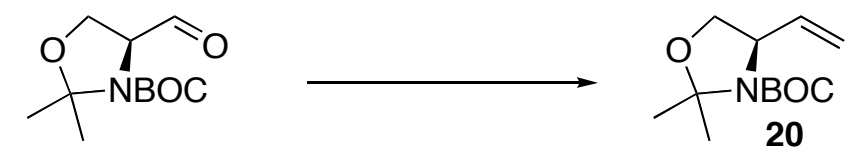

(4R)-2,2-Dimethyl-4-vinyloxazolidine-3-carboxylic acid tert-butyl ester (20). The title compound was prepared from (4S)-4-formyl-2,2-dimethyl-oxazolidine-3-carboxylic acid tert-butyl $\operatorname{ester}^{25}$ (230 mg, $1.00 \mathrm{mmol}$; the enantiomeric excess was determined to be $92 \%$ (24:1) by chiral GC analysis (20\% Permethylated G-Cyclodextrin, isothermal $70{ }^{\circ} \mathrm{C}, \mathrm{t}_{\mathrm{r}} 22,3$ (minor), 24.5 (major)). according to the general procedure A (reaction time: $4 \mathrm{~h}$ ). The desired alkene $\mathbf{2 0}(195 \mathrm{mg}, 86 \%)$ was obtained as a colorless oil after flash chromatography $\left(5 \% \mathrm{EtOAc} /\right.$ hexanes). $\mathrm{R}_{f} 0.28(5 \%$ EtOAc/hexanes); $[\square]^{25}=+12.5\left(\mathrm{c} 2.5, \mathrm{CHCl}_{3}\right) ;{ }^{1} \mathrm{H}$ NMR $\left(400 \mathrm{MHz}, \mathrm{C}_{6} \mathrm{D}_{6}\right) \square$ 5.85-5.82 (m (br), $1 \mathrm{H}), 5.22-5.11(\mathrm{~m}(\mathrm{br}), 1 \mathrm{H}), 5.08(\mathrm{~d}, J=10 \mathrm{~Hz}, 1 \mathrm{H}), 4.31-4.14(\mathrm{~m}(\mathrm{br}), 1 \mathrm{H}), 3.79$ (dd, $J=9,6 \mathrm{~Hz}$, $1 \mathrm{H}), 3.59$ (dd, $J=9,2 \mathrm{~Hz}, 1 \mathrm{H}), 1.81$ (s (br), 3H), 1.66 (s (br), 3H), $1.52(\mathrm{~s}, 9 \mathrm{H}) ;{ }^{13} \mathrm{C}$ NMR $(100$ $\left.\mathrm{MHz}, \mathrm{CDCl}_{3}\right) \square 151.8,137.2,136.6,115.5,93.7,80.0,79.3,67.9$, 59.5, 28.2 , 27.0, 26.3, 24.6, 23.5; IR (NaCl, film) 2980, 2940, 2870, 1690, 1375, 1255, 1175, 1090, 1060, 920, 860, 840, $770 \mathrm{~cm}^{-1}$; HMRS (MAB) calcd for $\mathrm{C}_{12} \mathrm{H}_{20} \mathrm{NO}_{3}\left[\mathrm{M}-\mathrm{H}^{+}\right]^{+}:$226.144319. Found: 226.143815. The enantiomeric excess was determined to be $92 \%(24: 1)$ by chiral GC analysis (20\% Permethylated GCyclodextrin, isothermal $70{ }^{\circ} \mathrm{C}, \mathrm{t}_{\mathrm{r}} 46.4$ (minor), 47.4 (major)).

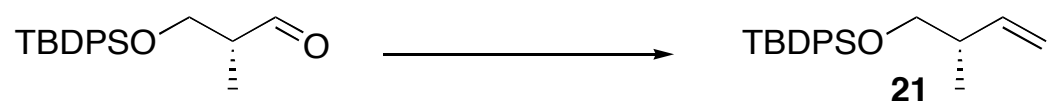

(S)-4-[(tert-Butyldiphenylsilyl)oxy]-3-methylbut-1-ene (21). ${ }^{26}$ The title compound was prepared from $(R)-3-\left[\left(\right.\right.$ tert-butyldiphenylsilyl)oxy]-2-methylpropanal $\left(163 \mathrm{mg}, 1.00 \mathrm{mmol} ;[\square]_{\mathrm{D}}{ }^{25}=-9.4(\mathrm{c}\right.$ 1.08, $\left.\mathrm{CHCl}_{3}\right)$; $\left(\right.$ lit. $^{26}[\square]_{\mathrm{D}}{ }^{25}=+4.0\left(\mathrm{c} 1.28, \mathrm{CHCl}_{3}\right)$ ) according to the general procedure $\mathbf{A}$ (reaction time: $1 \mathrm{~h})$. The desired alkene $21(117 \mathrm{mg}, 72 \%)$ was obtained as a colorless oil after flash chromatography (1\% EtOAc/hexanes); $\mathrm{R}_{f} 0.55$ (1\% EtOAc/hexanes); $[\square]_{\mathrm{D}}{ }^{25}=-3.18(c 0.71$, $\left.\mathrm{CHCl}_{3}\right) ;{ }^{1} \mathrm{H} \mathrm{NMR}\left(300 \mathrm{MHz}, \mathrm{CDCl}_{3}\right) \square 7.69-7.66(\mathrm{~m}, 4 \mathrm{H}), 7.43-7.38(\mathrm{~m}, 6 \mathrm{H}), 5.81$ (ddd, $J=14,9$, $7 \mathrm{~Hz}, 1 \mathrm{H}), 5.01(\mathrm{~m}, 2 \mathrm{H}), 3.57(\mathrm{dd}, J=9,6 \mathrm{~Hz}, 1 \mathrm{H}), 3.49(\mathrm{dd}, J=9,6 \mathrm{~Hz}, 1 \mathrm{H}), 2.40(\mathrm{~m}, 1 \mathrm{H}), 1.06$ $(\mathrm{s}, 9 \mathrm{H}), 1.04(\mathrm{~d}, J=7 \mathrm{~Hz}, 3 \mathrm{H}) ;{ }^{13} \mathrm{C}$ NMR $\left(75 \mathrm{MHz}, \mathrm{CDCl}_{3}\right) \square 141.2,135.5,133.8,129.4,127.4$, $113.9,68.4,40.1,26.7,19.2,16.0$. The enantiomeric excess was determined to be $94 \%(30: 1)$ by chiral GC analysis $\left(20 \%\right.$ Permethylated G-Cyclodextrin, $40{ }^{\circ} \mathrm{C}, 3 \mathrm{~min} ; 10{ }^{\circ} \mathrm{C} / \mathrm{min}, 230{ }^{\circ} \mathrm{C}, \mathrm{t}_{\mathrm{r}} 4.23$ (major), 4.36 (minor)) of the corresponding alcohol product after treatment of the alkene 21 with $\operatorname{TBAF}(1 \mathrm{M}$ in THF).

(S)-4-[(tert-Butyldiphenylsilyl)oxy]-3-methylbut-1-ene (21). ${ }^{26}$ The title compound was prepared from $(R)-3-\left[\left(\right.\right.$ tert-butyldiphenylsilyl)oxy]-2-methylpropanal $\left(163 \mathrm{mg}, 1.00 \mathrm{mmol} ;[\square]_{\mathrm{D}}^{25}=-9.4(\mathrm{c}\right.$ 1.08, $\left.\mathrm{CHCl}_{3}\right) ;\left(\right.$ lit. $^{26}[\square]_{\mathrm{D}}{ }^{25}=+4.0\left(\mathrm{c} 1.28, \mathrm{CHCl}_{3}\right)$ ) according to the general procedure $\mathbf{C}$ (reaction time: $2 \mathrm{~h}$ ). The desired alkene $21(119 \mathrm{mg}, 73 \%)$ was obtained as a colorless oil after flash chromatography $\left(1 \%\right.$ EtOAc/hexanes); $[\square]_{\mathrm{D}}{ }^{25}=-3.07\left(c 0.75, \mathrm{CHCl}_{3}\right)$; The enantiomeric excess was determined to be $90 \%(20: 1)$ by chiral GC analysis (20\% Permethylated G-Cyclodextrin, $40{ }^{\circ} \mathrm{C}, 3$ $\min ; 10{ }^{\circ} \mathrm{C} / \mathrm{min}, 230{ }^{\circ} \mathrm{C}, \mathrm{t}_{\mathrm{r}} 4.23$ (major), 4.36 (minor)) of the corresponding alcohol product after treatment of the alkene 21 with TBAF (1 M in THF).

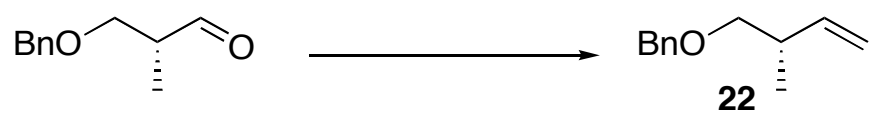

25 Avenoza, A.; Cativiela, C.; Corzana, F.; Peregrina, J. M.; Zurbano, M. M. J. Org. Chem. 1999, 64, 8220-8225.

26 Konno, K.; Fujishima, T.; Maki, S.; Liu, Z.; Miura, D.; Chokki, M.; Ishizuka, S.; Yamaguchi, K.; Kan, Y.; Kurihara, M.; Miyata, N.; Smith, C.; DeLuca, H. F.; Takayama, H. J. Med. Chem. 2000, 43, 4247-4265. 
(S)-(2-Methyl-but-3-enyloxymethyl)-benzene (22).27 The title compound was prepared from $(R)$ 3-benzyloxy-2-methylpropanal ${ }^{28},\left(178 \mathrm{mg}, 1.00 \mathrm{mmol}\right.$; $[\square]_{\mathrm{D}}{ }^{25}=-29.4$ (c 1.13, $\left.\mathrm{CHCl}_{3}\right)$; $\left(\right.$ lit. $^{28}[\square]_{\mathrm{D}}{ }^{25}=$ $+30.6\left(\mathrm{c} \mathrm{1.32}, \mathrm{CHCl}_{3}\right)$ ) according to the general procedure $\mathbf{A}$ (reaction time $3 \mathrm{~h}$ ). The desired alkene $22(137 \mathrm{mg}, 78 \%)$ was obtained as a colorless oil after flash chromatography (1\% EtOAc/hexanes). $\mathrm{R}_{f} 0.36(1 \% \mathrm{EtOAc} /$ hexanes$) ;[\square]_{\mathrm{D}}{ }^{25}=-3.0\left(c 1.0, \mathrm{CHCl}_{3}\right) ;{ }^{1} \mathrm{H} \mathrm{NMR}\left(400 \mathrm{MHz}, \mathrm{CDCl}_{3}\right) \square 7.36-7.26$ $(\mathrm{m}, 5 \mathrm{H}), 5.87-5.76(\mathrm{~m}, 1 \mathrm{H}), 5.11-4.99(\mathrm{~m}, 2 \mathrm{H}), 4.53(\mathrm{~s}, 2 \mathrm{H}), 3.42-3.28(\mathrm{~m}, 2 \mathrm{H}), 2.56-2.45(\mathrm{~m}, 1 \mathrm{H})$, $1.05(\mathrm{~d}, J=7 \mathrm{~Hz}, 3 \mathrm{H}) ;{ }^{13} \mathrm{C} \mathrm{NMR}\left(100 \mathrm{MHz}, \mathrm{CDCl}_{3}\right) \square 141.7,129.3,128.8,128.0,127.9,114.5$, 75.4, 73.4, 38.3, 17.0; The enantiomeric excess was determined to be $84 \%$ (12:1) by chiral HPLC analysis (Chiracel OJ, 1mL/min, 2\% 2-propanol/hexane, $\mathrm{t}_{\mathrm{r}} 59.3$ (major), 62.7 (minor), 68.2 (major), 85.9 (minor)) of the corresponding diol product.

(S)-(2-Methyl-but-3-enyloxymethyl)-benzene (22). The title compound was prepared from $(R)$-3benzyloxy-2-methylpropanal $\left(178 \mathrm{mg}, 1.00 \mathrm{mmol}\right.$; $[\square]_{\mathrm{D}}{ }^{25}=-29.4\left(\mathrm{c} 1.13, \mathrm{CHCl}_{3}\right) ;\left(\mathrm{lit}^{28}[\square]_{\mathrm{D}}{ }^{25}=\right.$ $+30.6\left(\mathrm{c} \mathrm{1.32}, \mathrm{CHCl}_{3}\right)$ ) according to the general procedure $\mathbf{B}$ (reaction time: $5 \mathrm{~h}$ ). The desired alkene 22 (134 mg, 76\%) was obtained as a colorless oil after flash chromatography (1\% EtOAc/hexanes). $\mathrm{R}_{f} 0.36$ ( $1 \%$ EtOAc/hexanes); The enantiomeric excess was determined to be $79 \%$ (8:1) by chiral HPLC analysis, (Chiracel OJ, $1 \mathrm{~mL} / \mathrm{min}, 2 \%$ 2-propanol/hexane, $\mathrm{t}_{\mathrm{r}} 59.3$ (major), 62.7 (minor), 68.2 (major), 85.9 (minor)) of the corresponding diol product.

(S)-(2-Methyl-but-3-enyloxymethyl)-benzene (22). The title compound was prepared from (R)-3benzyloxy-2-methylpropanal $(50.0 \mathrm{mg}, 0.28 \mathrm{mmol})$ according to the general procedure $\mathbf{C}$ (reaction time $2 \mathrm{~h}$ ). The desired alkene 22 (26 mg, 53\%) was obtained as a colorless oil after flash chromatography ( $1 \%$ EtOAc/hexanes). $\mathrm{R}_{f} 0.36$ (1\% EtOAc/hexanes); The enantiomeric excess was determined to be $80 \%(8: 1)$ by chiral HPLC analysis, (Chiracel OJ, $1 \mathrm{~mL} / \mathrm{min}, 2 \%$ 2propanol/hexane, $\mathrm{t}_{\mathrm{r}} 59.3$ (major), 62.7 (minor), 68.2 (major), 85.9 (minor)) of the corresponding diol product.<smiles>CC(CO)C(O)CO</smiles>

4-Benzyloxy-3-methyl-butane-1,2-diol. The isolated alkene 22 from procedure $\mathbf{A}$ was treated with AD-mix alpha in $t$ - $\mathrm{BuOH} / \mathrm{H}_{2} \mathrm{O}$ for $48 \mathrm{~h} .{ }^{29}$ The desired diol was isolated as a mixture of diastereoisomers and as a colorless oil after flash chromatography (50\% EtOAc/hexanes). $\mathrm{R}_{f} 0.24$ $\left(50 \%\right.$ EtOAc/hexanes); [ []$_{\mathrm{D}}{ }^{25}=-11.0\left(c 1.0, \mathrm{CHCl}_{3}\right)$; Major diastereoisomer: ${ }^{1} \mathrm{H}$ NMR $(400 \mathrm{MHz}$, $\left.\mathrm{CDCl}_{3}\right) \square 7.38-7.28(\mathrm{~m}, 5 \mathrm{H}), 4.53(\mathrm{~s}, 2 \mathrm{H}), 3.76-3.43(\mathrm{~m}, 5 \mathrm{H}), 2.81-2.76(\mathrm{~s}(\mathrm{br}), 2 \mathrm{H}), 2.06-1.95$ (m, $1 \mathrm{H}), 0.90(\mathrm{~d}, J=7 \mathrm{~Hz}, 3 \mathrm{H}) ;{ }^{13} \mathrm{C}$ NMR $\left(100 \mathrm{MHz}, \mathrm{CDCl}_{3}\right) \square 137.3,128.4,127.8,127.7,127.6,76.1$, 74.5, 74.4, 73.4, 64.5, 36.0, 13.8; IR (neat) 3370 (br), 3030, 2860, 1497, 1454, 1364, 1073, 699, $631 \mathrm{~cm}^{-1}$; HMRS (MAB) calcd for $\mathrm{C}_{12} \mathrm{H}_{18} \mathrm{O}_{3}\left[\mathrm{M}+\mathrm{H}^{+}\right]^{+}:$211.13342. Found: 211.13280 .<smiles>O=CC(OCc1ccccc1)C(OCc1ccccc1)C(C=O)C(Br)(Br)c1ccccc1</smiles><smiles>C=CC(OCc1ccccc1)C(OCc1ccccc1)C(CC(C)C)C(Br)(Br)c1ccccc1</smiles>

(3R, 4R, 5R, 6S)-3,4,5,6-Tetra(benzyloxy)-1,7-octadiene (23). ${ }^{30}$ The title compound was prepared from 2,3,4,5,-tetra-(benzyloxy)-1,6-hexanedial $(50.0 \mathrm{mg}, 0.10 \mathrm{mmol})$ and 2-methyl-1-

27 Grandguillot, J. C.; Rouessac, F. Tetrahedron 1991, 47, 5133-5148.

28 Kawabata, T.; Kimura, Y.; Ito, Y.; Terashima, S.; Sasaki, A.; Sunagawa, M. Tetrahedron 1988, 44, $2149-2165$.

29 Kolb, H. C.; Andersson, P. G.; Sharpless, K. B. J. Am. Chem. Soc. 1994, 116, 1278-1291.

30 Ackermann, L.; El Tom, D.; Furstner, A. Tetrahedron 2000, 56, 2195-2202. 
propanol $(0.2 \mathrm{~mL})$ according to the general procedure $\mathbf{A}$ (reaction time: $2 \mathrm{~h}$ ). The desired diene $\mathbf{2 3}$ (24 mg, 50\%) was obtained as a colorless oil after flash chromatography (2\% EtOAc/hexanes). $\mathrm{R}_{f}$ $0.22(2 \% \text { EtOAc/hexanes); [ }]_{\mathrm{D}}{ }^{25}=+13.0\left(c\right.$ 1.0, $\left.\mathrm{CHCl}_{3}\right) ;{ }^{1} \mathrm{H} \mathrm{NMR}\left(400 \mathrm{MHz}, \mathrm{CDCl}_{3}\right) \square 7.32-7.24$ $(\mathrm{m}, 20 \mathrm{H}), 5.99-5.81(\mathrm{~m}, 2 \mathrm{H}), 5.35-5.18(\mathrm{~m}, 4 \mathrm{H}), 4.82-4.50(\mathrm{~m}, 6 \mathrm{H}), 4.36-3.70(\mathrm{~m}, 6 \mathrm{H}) ;{ }^{13} \mathrm{C}$ NMR $\left(75 \mathrm{MHz}, \mathrm{CDCl}_{3}\right) \square 139.4,139.3,138.9,138.7,136.6,136.0,128.7,128.6,128.5,128.3,128.1$, $128.0,127.9,128.7,120.1,119.5,81.7,81.5,80.9,80.8,75.6,74.4,70.8,70.3$.<smiles>O=Cc1ccc2c(c1)OCO2</smiles>
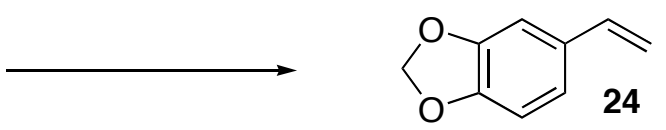

5-Vinylbenzo[1,3]dioxole (24). The title compound was prepared from piperonal $(75 \mathrm{mg}, 0.50$ mmol) according to the general procedure A (reaction time: $1 \mathrm{~h}$ ). The desired alkene $24(55 \mathrm{mg}$, $74 \%$ ) was obtained as a colorless oil after flash chromatography (2\% EtOAc/hexanes). $\mathrm{R}_{f} 0.37$ (2\% EtOAc/hexanes); ${ }^{1} \mathrm{H}$ NMR $\left(400 \mathrm{MHz}, \mathrm{CDCl}_{3}\right) \square 6.97(\mathrm{~s}, 1 \mathrm{H}), 6.84(\mathrm{~d}, J=8 \mathrm{~Hz}, 1 \mathrm{H}), 6.77(\mathrm{~d}, J=8$ $\mathrm{Hz}, 1 \mathrm{H}), 6.63(\mathrm{dd}, J=18,11 \mathrm{~Hz}, 1 \mathrm{H}), 5.96(\mathrm{~s}, 2 \mathrm{H}), 5.58(\mathrm{~d}, J=18 \mathrm{~Hz}, 1 \mathrm{H}), 5.13(\mathrm{~d}, J=11 \mathrm{~Hz}$, $1 \mathrm{H}) ;{ }^{13} \mathrm{C} \mathrm{NMR}\left(100 \mathrm{MHz}, \mathrm{CDCl}_{3}\right) \square 147.8,147.2,136.2,132.0,120.9,111.8,108.0,105.2,100.9$; IR (neat) 3087, 3008, 2981, 2893, 1630, 1604, 1504, 1444, 1350, 1247, 1043, 939, 915, $813 \mathrm{~cm}^{-1}$; Anal. Calcd for $\mathrm{C}_{9} \mathrm{H}_{8} \mathrm{O}_{2}$ : C, 72.96; H, 5.44. Found: C, 72.94; H, 5.49.

5-Vinylbenzo[1,3]dioxole (24). The title compound was prepared from piperonal $(150 \mathrm{mg}, 1.00$ mmol) according to the general procedure $\mathbf{B}$ (reaction time: $2 \mathrm{~h}$ ). The desired alkene $\mathbf{2 4}$ (120 $\mathrm{mg}$, $81 \%$ ) was obtained as a colorless oil after flash chromatography (2\% EtOAc/hexanes).

5-Vinylbenzo[1,3]dioxole (24). The title compound was prepared from piperonal (150 $\mathrm{mg}, 1.00$ $\mathrm{mmol}$ ) according to the general procedure $\mathbf{C}$ (reaction time: $3 \mathrm{~h}$ ). The desired alkene $\mathbf{2 4}$ (125 $\mathrm{mg}$, $84 \%$ ) was obtained as a colorless oil after flash chromatography (2\% EtOAc/hexanes).<smiles>O=Cc1ccc(Br)cc1</smiles>

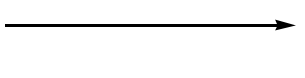<smiles>C=Cc1ccc(Br)cc1</smiles>

1-Bromo-4-vinylbenzene (25). The title compound was prepared from 4-bromo-benzaldehyde (183 $\mathrm{mg}, 1.00 \mathrm{mmol}$ ) according to the general procedure $\mathbf{A}$ (reaction time: $1 \mathrm{~h}$ ). The desired alkene 25 (120 mg, 66\%) was obtained as a colorless oil after flash chromatography (1\% ether/pentane); ${ }^{1} \mathrm{H} \mathrm{NMR}\left(400 \mathrm{MHz}, \mathrm{CDCl}_{3}\right) \square 7.46(\mathrm{~d}, J=8 \mathrm{~Hz}, 2 \mathrm{H}), 7.28(\mathrm{~d}, J=8 \mathrm{~Hz}, 2 \mathrm{H}), 6.67(\mathrm{dd}, J=18,11$ $\mathrm{Hz}, 1 \mathrm{H}), 5.76(\mathrm{~d}, J=18 \mathrm{~Hz}, 1 \mathrm{H}), 5.29(\mathrm{~d}, J=11 \mathrm{~Hz}, 1 \mathrm{H})$.

1-Bromo-4-vinylbenzene (25). The title compound was prepared from 4-bromo-benzaldehyde (183 $\mathrm{mg}, 1.00 \mathrm{mmol}$ ) according to the general procedure $\mathbf{B}$ (reaction time $2 \mathrm{~h}$ ). The desired alkene 25 (140 mg, 77\%) was obtained as a colorless oil after flash chromatography (1\% ether/pentane).<smiles>COc1ccc(C=O)cc1</smiles>
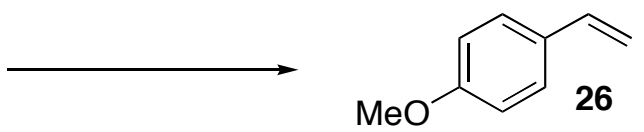

1-Methoxy-4-vinylbenzene (26). The title compound was prepared from 4-methoxy-benzaldehyde $(136 \mathrm{mg}, 1.00 \mathrm{mmol})$ according to the general procedure A (reaction time: $0.5 \mathrm{~h}$ ). The desired alkene 26 (107 mg, 80\%) was obtained as a colorless oil after flash chromatography (1\% ether/pentane); ${ }^{1} \mathrm{H}$ NMR (400 MHz, $\left.\mathrm{CDCl}_{3}\right) \square 7.36(\mathrm{~d}, J=9 \mathrm{~Hz}, 1 \mathrm{H}), 6.88(\mathrm{~d}, J=9 \mathrm{~Hz}, 1 \mathrm{H}), 6.69$ $(\mathrm{dd}, J=18,11 \mathrm{~Hz}, 1 \mathrm{H}), 5.62(\mathrm{~d}, J=18 \mathrm{~Hz}, 1 \mathrm{H}), 5.14(\mathrm{~d}, J=11 \mathrm{~Hz}, 1 \mathrm{H}), 3.82(\mathrm{~s}, 3 \mathrm{H})$. 
1-Methoxy-4-vinylbenzene (26). The title compound was prepared from 4-methoxy-benzaldehyde (136 $\mathrm{mg}, 1.00 \mathrm{mmol}$ ) according to the general procedure $\mathbf{B}$ (reaction time: $0.5 \mathrm{~h}$ ). The desired alkene 26 (120 mg, 90\%) was obtained as a colorless oil after flash chromatography (1\% ether/pentane).

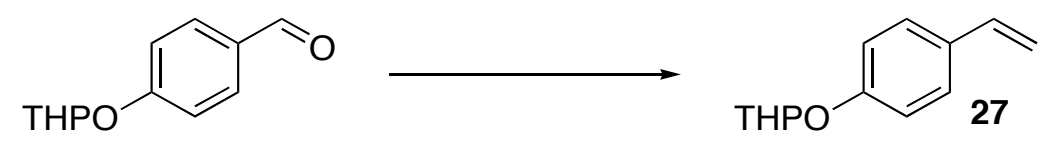

1-(Tetrahydropyran-2-yloxy)-4-vinylbenzene (27). ${ }^{31}$ The title compound was prepared from 4(tetrahydropyran-2-yloxy)benzaldehyde $(206 \mathrm{mg}, 1.00 \mathrm{mmol})$ according to the general procedure A (reaction time: $1 \mathrm{~h})$. The desired alkene $27(190 \mathrm{mg}, 93 \%)$ was obtained as a colorless oil after flash chromatography (2\% EtOAc/hexanes); $\mathrm{R}_{f} 0.42$ (2\% EtOAc/hexanes); ${ }^{1} \mathrm{H}$ NMR $\left(400 \mathrm{MHz}, \mathrm{CDCl}_{3}\right)$ $\square 7.36(\mathrm{~d}, J=9 \mathrm{~Hz}, 2 \mathrm{H}), 7.03(\mathrm{~d}, J=9 \mathrm{~Hz}, 2 \mathrm{H}), 6.68(\mathrm{dd}, J=18,11 \mathrm{~Hz}, 1 \mathrm{H}), 5.64(\mathrm{dd}, J=18,1$ $\mathrm{Hz}, 1 \mathrm{H}), 5.44(\mathrm{t}, J=3 \mathrm{~Hz}, 1 \mathrm{H}), 5.15(\mathrm{dd}, J=11,1 \mathrm{~Hz}, 1 \mathrm{H}), 3.92(\mathrm{dd}, J=10,3 \mathrm{~Hz}, 1 \mathrm{H}), 3.63-3.60$ $(\mathrm{m}, 1 \mathrm{H}), 2.03-2.00(\mathrm{~m}, 1 \mathrm{H}), 1.90-1.86(\mathrm{~m}, 2 \mathrm{H}), 1.72-1.60(\mathrm{~m}, 3 \mathrm{H}) ;{ }^{13} \mathrm{C} \mathrm{NMR}\left(100 \mathrm{MHz}, \mathrm{CDCl}_{3}\right) \square$ $156.7,136.1,131.1,127.1,116.3,111.7,96.1,61.8,30.2,25.1,18.6$.

1-(Tetrahydropyran-2-yloxy)-4-vinylbenzene (27). The title compound was prepared from 4(tetrahydropyran-2-yloxy)benzaldehyde $(206 \mathrm{mg}, 1.00 \mathrm{mmol}$ ) according to the general procedure B (reaction time: $3 \mathrm{~h}$ ). The desired alkene $27(165 \mathrm{mg}, 81 \%)$ was obtained as a colorless oil after flash chromatography (2\% EtOAc/hexanes).<smiles>CCCCCCCCNCCc1cc(OC)c(OC)cc1C=O</smiles><smiles>C=Cc1cc(OC)c(OC)cc1CCNCCCCC</smiles>

$N$-[2-(4,5-Dimethoxy-2-vinylphenyl)ethyl]acetamide (28). The title compound was prepared from $N$-[2-(2-formyl-4,5-dimethoxy-phenyl)-ethyl]-acetamide ${ }^{32}(390 \mathrm{mg}, 1.55 \mathrm{mmol})$ according to the general procedure A (reaction time: $0.5 \mathrm{~h})$. The desired alkene $\mathbf{2 8}(233 \mathrm{mg}, 60 \%)$ was obtained as a yellow solid after flash chromatography (20\% DCM/acetone with $1 \%$ triethylamine) and recristallisation in TBME. $\mathrm{R}_{f} 0.27$ (2\% EtOAc/hexanes); ${ }^{1} \mathrm{H}$ NMR (400 MHz, $\left.\mathrm{CDCl}_{3}\right) \square 7.03$ (s, $1 \mathrm{H}), 6.93$ (dd, $J=17,11 \mathrm{~Hz}, 1 \mathrm{H}), 6.64(\mathrm{~s}, 1 \mathrm{H}), 5.57$ (d, $J=17 \mathrm{~Hz}, 1 \mathrm{H}), 5.49$ (s(br), 1H), 5.24 (d, $J$ $=11 \mathrm{~Hz}, 1 \mathrm{H}), 3.90(\mathrm{~s}, 3 \mathrm{H}), 3.87(\mathrm{~s}, 3 \mathrm{H}), 3.43(\mathrm{q}, J=7 \mathrm{~Hz}, 2 \mathrm{H}), 2.85(\mathrm{t}, J=7 \mathrm{~Hz}, 2 \mathrm{H}), 1.93(\mathrm{~s}, 3 \mathrm{H})$; ${ }^{13} \mathrm{C}$ NMR (75 MHz, $\left.\mathrm{CDCl}_{3}\right) \square 170.5,149.3,148.2,134.2,129.5,129.46,114.3,113.1,108.9,56.37$, 56.31, 41.1, 32.7, 23.8; IR (HATR, solid) 3245, 3075, 2935, 1630, 1510, 1460, 1260, 1220, 1100, 985, $865 \mathrm{~cm}^{-1}$; Anal. Calcd for $\mathrm{C}_{14} \mathrm{H}_{19} \mathrm{NO}_{3}: \mathrm{C}, 67.45 ; \mathrm{H}, 7.68 ; \mathrm{N}, 5.62$. Found: $\mathrm{C}, 66.44 ; \mathrm{H}, 7.45$; N, 5.46 .

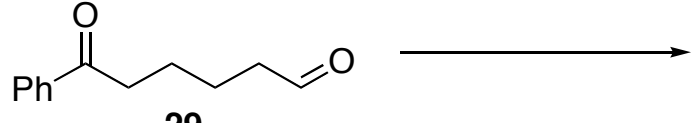

29<smiles>C=CCCCCC(=O)c1ccccc1</smiles>

30

1-Phenylhept-6-en-1-one (30). The title compound was prepared from 6-oxo-6-phenylhexanal (29) $(630 \mathrm{mg}, 3.31 \mathrm{mmol})$ according to the general procedure $\mathbf{A}$ (reaction time: $0.5 \mathrm{~h}$ ). The desired alkene 30 (542 mg, 87\%) was obtained as a colorless oil after flash chromatography (2\% EtOAc/hexanes); $\mathrm{R}_{f} 0.27$ (2\% EtOAc/hexanes); ${ }^{1} \mathrm{H} \mathrm{NMR}\left(400 \mathrm{MHz}, \mathrm{CDCl}_{3}\right) \square$ 7.97-7.95 (m, 2H),

31 Srikrishna, A.; Sattigeri, J. A.; Viswajanani, R.; Yelamaggad, C. V. J. Org. Chem. 1995, 60, 2260-2260.

32 Wirth, T.; Fragale, G. Synthesis 1998, 162-166. 
7.57-7.54 (m, 1H), 7.48-7.44 (m, 2H), 5.86-5.79 (m, 1H), 5.05-4.95 (m, 2H), $2.98(\mathrm{t}, J=7 \mathrm{~Hz}, 2 \mathrm{H})$, 2.14-2.08 (m, 2H), 1.80-1.73 (m, 2H), 1.53-1.47 (m, 2H); ${ }^{13} \mathrm{C}$ NMR (100 MHz, CDCl $\left.\mathrm{Cl}_{3}\right) 200.2$, 138.4, 136.9, 132.8, 128.4, 127.9, 114.5, 38.3, 33.4, 28.4, 23.6; IR (NaCl, film) 3065, 2935, 2860, 1690, 1450, 1225, 910, $690 \mathrm{~cm}^{-1}$; Anal. Calcd for $\mathrm{C}_{13} \mathrm{H}_{16} \mathrm{O}: \mathrm{C}, 82.94 ; \mathrm{H}, 8.57$. Found: $\mathrm{C}, 82.97$; $\mathrm{H}$, 8.55. HMRS (MAB) calcd for $\mathrm{C}_{13} \mathrm{H}_{16} \mathrm{O}[\mathrm{M}]^{+}$: 188.120115. Found: 188.119597.

1-Phenylhept-6-en-1-one (30). The title compound was prepared from 6-oxo-6-phenylhexanal (29) $(190 \mathrm{mg}, 1.00 \mathrm{mmol}$ ) according to the general procedure $\mathbf{C}$ (reaction time: $3 \mathrm{~h}$ ). The desired alkene $30(111 \mathrm{mg}, 59 \%)$ was obtained as a colorless oil after flash chromatography (2\% EtOAc/hexanes).<smiles>OCCCCC(O)CO</smiles>

6-Benzyloxy-hexane-1,5-diol. To a solution of 1,2,6-hexanetriol (670 mg, $5.00 \mathrm{mmol})$ in $\mathrm{MeOH}$ $(50 \mathrm{~mL})$ was added dibutyltin oxide $(1.24 \mathrm{~g}, 5.00 \mathrm{mmol})$. After 2 hours at reflux, the reaction mixture was evaporated to dryness and the white solid was dissolved in benzene (100 $\mathrm{mL})$. Tetrabutylammonium iodide $(2.77 \mathrm{~g}, 7.50 \mathrm{mmol})$ and benzyl bromide $(0.65 \mathrm{~mL}, 5.50 \mathrm{mmol})$ were then added to the reaction mixture at room temperature. After 16 hours at $50{ }^{\circ} \mathrm{C}$, the solution was concentrated down. The desired diol $(0.96 \mathrm{~g}, 86 \%)$ was obtained as a colorless oil after flash chromatography (100\% EtOAc); $\mathrm{R}_{f} 0.39$ (100\% EtOAc); ${ }^{1} \mathrm{H}$ NMR $\left(300 \mathrm{MHz}, \mathrm{CDCl}_{3}\right) \square 7.35-7.29$ $(\mathrm{m}, 5 \mathrm{H}), 4.54(\mathrm{~s}, 2 \mathrm{H}), 3.82-3.79(\mathrm{~m}, 1 \mathrm{H}), 3.59(\mathrm{t}, J=6 \mathrm{~Hz}, 2 \mathrm{H}), 3.48(\mathrm{dd}, J=9,3 \mathrm{~Hz}, 1 \mathrm{H}), 3.33$ $(\mathrm{dd}, J=9,8 \mathrm{~Hz}, 1 \mathrm{H}), 2.88(\mathrm{br}, 1 \mathrm{H}), 2.36(\mathrm{br}, 1 \mathrm{H}), 1.58-1.39(\mathrm{~m}, 6 \mathrm{H}) ;{ }^{13} \mathrm{C} \mathrm{NMR}\left(75 \mathrm{MHz}, \mathrm{CDCl}_{3}\right) \square$ 137.8, 128.3, 127.6, 74.5, 73.2, 70.2, 62.3, 32.5, 32.3, 21.5; IR (neat) 3370 (br), 3343, 2934, 2861, 1454, 1364, 1092, 699, $631 \mathrm{~cm}^{-1}$; HMRS (FAB) calcd for $\mathrm{C}_{13} \mathrm{H}_{20} \mathrm{O}_{3}\left[\mathrm{M}+\mathrm{H}^{+}\right]^{+}: 225.149070$. Found: 225.148681 .<smiles>OCCCCC(O)COCc1ccccc1</smiles><smiles>O=CCCCC(=O)COc1ccccc1</smiles>

6-Benzyloxy-5-oxo-hexanal. (32). Oxalyl chloride $(44.0 \mu \mathrm{L}, 0.50 \mathrm{mmol})$ was added to a solution of dimethylsulfoxide $(71.0 \mu \mathrm{L}, 1.00 \mathrm{mmol})$ in dichloromethane $(4 \mathrm{~mL})$ at $-78{ }^{\circ} \mathrm{C}$. After $15 \mathrm{~min}$, a solution of the diol $(22.0 \mathrm{mg}, 0.10 \mathrm{mmol})$ in dichloromethane $(1 \mathrm{~mL})$ was added. The resulting mixture was stirred for $1 \mathrm{~h}$ at $-78^{\circ} \mathrm{C}$ before triethylamine $(0.27 \mathrm{~mL}, 2.00 \mathrm{mmol})$ was added. The cooling bath was then removed and the solution was stirred for another hour at room temperature. Saturated aqueous $\mathrm{NH}_{4} \mathrm{Cl}(15 \mathrm{~mL})$ was added and the aqueous layer was extracted with dichloromethane $(3 \times 20 \mathrm{~mL})$. The combined organic layers were washed with saturated aqueous $\mathrm{NaHCO}_{3}(15 \mathrm{~mL})$, brine $(15 \mathrm{~mL})$ and dried over $\mathrm{MgSO}_{4}$. After evaporation, the desired ketoaldehyde 32 (12 mg, 55\%) was obtained as a colorless oil after flash chromatography (1:2 EtOAc/hexanes); $\mathrm{R}_{f} 0.27$ (1:2 EtOAc/hexanes); ${ }^{1} \mathrm{H}$ NMR (300 MHz, $\left.\mathrm{C}_{6} \mathrm{D}_{6}\right) \square 9.22(\mathrm{~s}, 1 \mathrm{H}), 7.24-$ $7.10(\mathrm{~m}, 5 \mathrm{H}), 4.22(\mathrm{~s}, 2 \mathrm{H}), 3.61(\mathrm{~s}, 2 \mathrm{H}), 2.05(\mathrm{t}, J=7 \mathrm{~Hz}, 2 \mathrm{H}), 1.79(\mathrm{t}, J=7 \mathrm{~Hz}, 2 \mathrm{H}), 1.62-1.58(\mathrm{~m}$, $2 \mathrm{H}) ;{ }^{13} \mathrm{C}$ NMR (100 MHz, $\left.\mathrm{C}_{6} \mathrm{D}_{6}\right) \square 204.2,197.4,125.8,125.4,125.2,125.0,72.4,70.4,40.0,34.8$, 12.8; IR (neat) 3031, 2865, 1717, 1455, 1391, 1099, 739, 699, $602 \mathrm{~cm}^{-1}$; HMRS (FAB) calcd for $\mathrm{C}_{13} \mathrm{H}_{16} \mathrm{O}_{3}\left[\mathrm{M}-\mathrm{H}^{+}\right]^{+}:$219.102120. Found: 219.102261 .
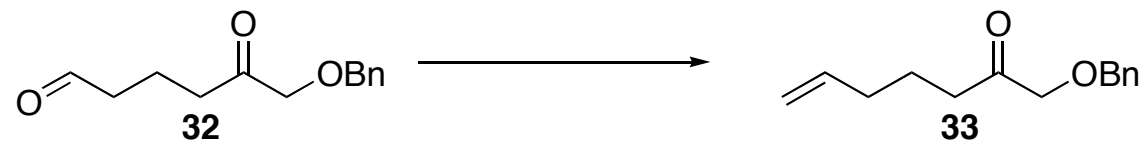
6-Benzyloxy-hept-6-en-2-one. (33). The title compound was prepared from keto-aldehyde 32 (163 $\mathrm{mg}, 1.00 \mathrm{mmol}$ ) according to the general procedure $\mathbf{A}$ (reaction time: $1 \mathrm{~h}$ ). The desired alkene $\mathbf{3 3}$ (117 mg, 72\%) was obtained as a colorless oil after flash chromatography (5\% EtOAc/hexanes); $\mathbf{R}_{f}$ 0.18 (5\% EtOAc/hexanes); ${ }^{1} \mathrm{H}$ NMR (300 MHz, $\left.\mathrm{CDCl}_{3}\right) \square 7.37-7.33(\mathrm{~m}, 5 \mathrm{H}), 5.81-5.70(\mathrm{~m}, 1 \mathrm{H})$, 5.05-4.96 (m, 2H), 4.59 (s, 2H), $4.06(\mathrm{~s}, 2 \mathrm{H}), 2.47(\mathrm{t}, J=7 \mathrm{~Hz}, 2 \mathrm{H}), 2.10-2.03(\mathrm{~m}, 2 \mathrm{H}), 1.73-1.68$ $(\mathrm{m}, 2 \mathrm{H}) ;{ }^{13} \mathrm{C}$ NMR $\left(75 \mathrm{MHz}, \mathrm{CDCl}_{3}\right) \square 208.5,137.7,137.1,128.4,127.9,127.8,115.2,74.9,73.2$, $38.0,32.9,22.1$; IR (neat) 3067, 2933, 1721, 1455, 1097, 913, 698, $630 \mathrm{~cm}^{-1}$.

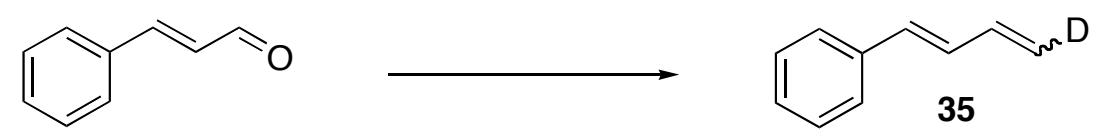

4-phenyl-(1D)-1,3-butadiene (35). The title compound was prepared from trans-cinnamaldehyde (250 $u \mathrm{~L}, 2.00 \mathrm{mmol})$ and 2-propan $(\mathrm{ol}-d)$ according to the general procedure $\mathbf{A}$ (reaction time: 0.5 h). The desired alkene 35 (180 mg, 69\%) was obtained as a colorless oil after flash chromatography (1\% ether/pentane); ${ }^{1} \mathrm{H}$ NMR $\left(400 \mathrm{MHz}, \mathrm{CDCl}_{3}\right) \square 7.46-7.44(\mathrm{~m}, 2 \mathrm{H}), 7.38-7.34(\mathrm{~m}, 2 \mathrm{H})$, 7.29-7.27 (m, 1H), $6.84(\mathrm{ddd}, J=16,10,1 \mathrm{~Hz}, 1 \mathrm{H}), 6.61(\mathrm{~d}, J=16 \mathrm{~Hz}, 1 \mathrm{H}), 6.60-6.51(\mathrm{~m}, 1 \mathrm{H}), 5.40-5.19(\mathrm{~m}$, $1 \mathrm{H}) ;{ }^{13} \mathrm{C} \mathrm{NMR}\left(100 \mathrm{MHz}, \mathrm{CDCl}_{3}\right) \square 137.1,137.0,132.7,129.5,128.5,127.5,126.3,117.5$; HMRS (MAB) calcd for $\mathrm{C}_{10} \mathrm{H}_{9} \mathrm{D}[\mathrm{M}]^{+}: 130.078250$. Found: 130.078195 . 
GC Trace of Various Trimethylsilyldiazomethane Solutions

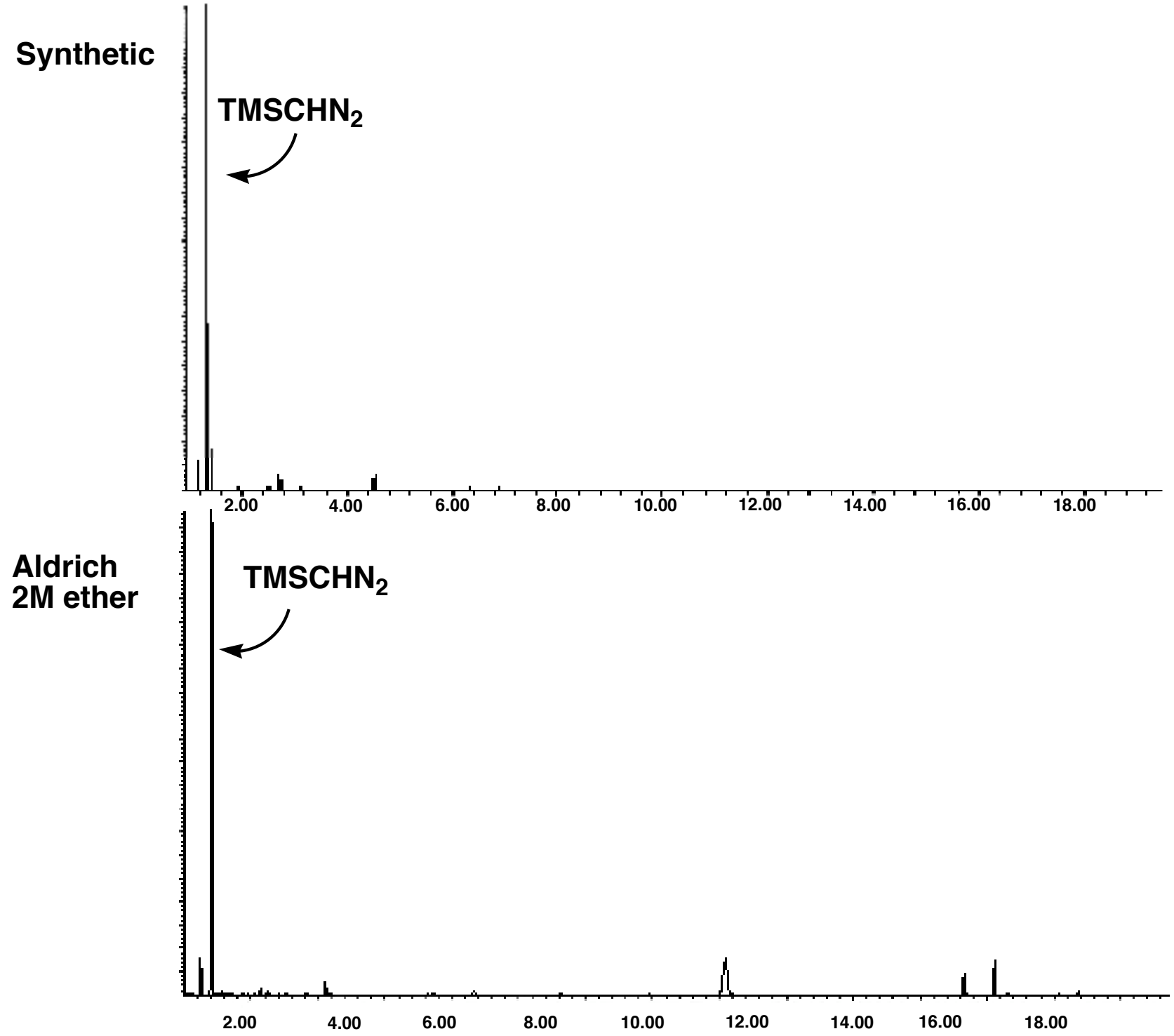


GC Trace of Various Trimethylsilyldiazomethane Solutions
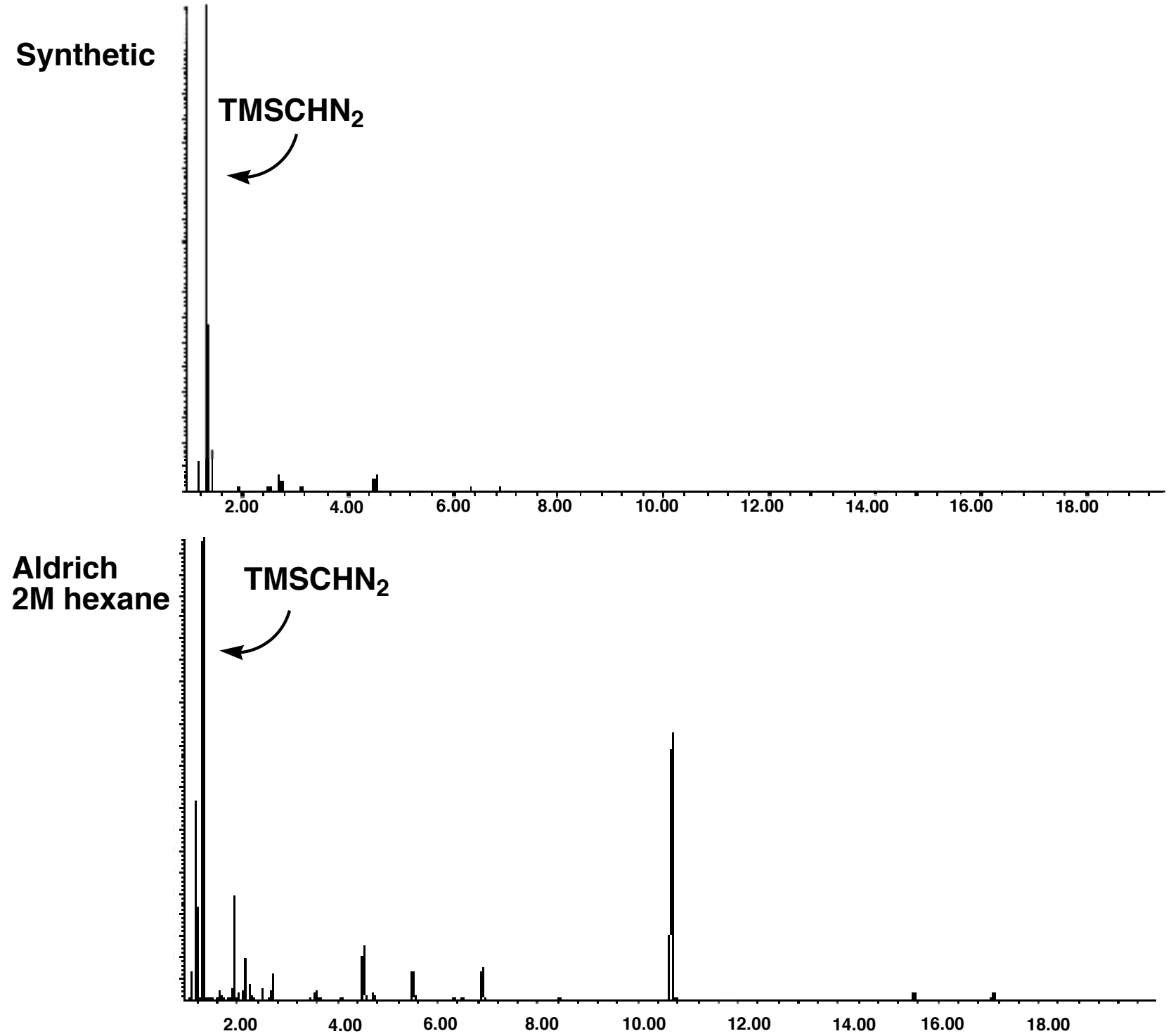